\title{
Synthesis of 2,3-Substituted Thienylboronic Acids
}

\section{and Esters}

Claus Christophersen ${ }^{*, *}$, Mikael Begtrup ${ }^{*}$,S $\phi r e n$ Ebdrup $^{\S}$, Henning Petersen ${ }^{\S}$ and Per Veds $\phi^{\S}$

Novo Nordisk A/S, Novo Allé, DK-2880 Bagsværd, Denmark and Department of Medicinal

Chemistry, The Danish University for Pharmaceutical Sciences, Universitetsparken 2, DK2200 Copenhagen N, Denmark

clcp@novonordisk.com

\section{Table of cotents.}

$\begin{array}{ll}\text { General Experimental Procedure } & \text { S3 }\end{array}$

3-Iodotiophene

S3-S4

Typical Procedure for the Preparation of 3-Bromo-2-thienylmagnesium

Chloride (2)

Typical Procedure for the Preparation of 2-Bromo-3-thienylmagnesium

Chloride (5)

Ethyl 3-Bromo-2-thiophenecarboxylate (7a)

$N$-tert-Butyl 3-Bromothienyl-2-carboxamide (7b)

3-Bromo-2-trimethylsilylthiophene (7c)

3-Acetyl-2-bromothiophene (8a)

2-Bromo-3-cyanothiophene $(\mathbf{8 b})$

2-Bromo-3-thienylcarbaldehyde (8c)

Ethyl 2-Bromo-3-thiophenecarboxylate (8d)

General Procedure for the Borylation of 2,3-Substituted Bromothiophenes with Pinacolborane Catalyzed by $\operatorname{Pd}\left(\mathrm{P}(t-\mathrm{Bu})_{3}\right)_{2}$ 
2-Carboxyethyl-3-thienylboronic Acid (9a)

S7-S8

$N$-tert-Butyl 3-(4,4,5,5-tetramethyl-1,3,2-dioxaborolan-2-yl)-2-thiophene

Carboxamide (9b)

S8

3-(4,4,5,5-Tetramethyl-1,3,2-dioxaborolan-2-yl)-3-trimethylsilylthiophene (9c) S8

2-Nitro-3-(4,4,5,5-tetramethyl-1,3,2-dioxaborolan-2-yl)-thiophene (9d) S9

3-Acetyl-2-thienylboronic Acid (10a) S9

2-(4,4,5,5-Tetramethyl-1,3,2-dioxaborolan-2-yl)-3-thienylcarbaldehyde (10c) S9

3-Carboxyethyl-2-thiopheneboronic Acid (10d) S10

$N$-tert-Butyl 3-(4-( $N^{\prime}, N^{\prime}$-dimethylamino)phenyl)-2-thiophene Carboxamide (11)S10-S11

References

S11-S12

${ }^{1} \mathrm{H}$ - and ${ }^{13} \mathrm{C}-\mathrm{NMR}$ spectra for Ethyl 3-Bromo-2-thiophenecarboxylate (7a) $\quad$ S13-S14

${ }^{1} \mathrm{H}$ - and ${ }^{13} \mathrm{C}$-NMR spectra for $\mathrm{N}$-tert-Butyl 3-Bromothienyl-2-carboxamide (7b) S15-S16

${ }^{1} \mathrm{H}$ - and ${ }^{13} \mathrm{C}$-NMR spectra for 3-Bromo-2-trimethylsilylthiophene (7c) $\quad$ S17-S18

${ }^{1} \mathrm{H}$ - and ${ }^{13} \mathrm{C}$-NMR spectra for 3-Acetyl-2-bromothiophene (8a) S19-S20

${ }^{1} \mathrm{H}$ - and ${ }^{13} \mathrm{C}-\mathrm{NMR}$ spectra for 2-Bromo-3-cyanothiophene $(\mathbf{8 b}) \quad$ S21-S22

${ }^{1} \mathrm{H}$ - and ${ }^{13} \mathrm{C}$-NMR spectra for 2-Bromo-3-thienylcarbaldehyde (8c) S23-S24

${ }^{1} \mathrm{H}$ - and ${ }^{13} \mathrm{C}-\mathrm{NMR}$ spectra for Ethyl 2-Bromo-3-thiophenecarboxylate (8d) $\quad$ S25-S26

${ }^{1} \mathrm{H}$ - and ${ }^{13} \mathrm{C}$-NMR spectra for 2-Carboxyethyl-3-thienylboronic Acid (9a) S27-S28

${ }^{1} \mathrm{H}$ - and ${ }^{13} \mathrm{C}$-NMR spectra for $N$-tert-Butyl 3-(4,4,5,5-tetramethyl-1,3,2-dioxaborolan-2-

$\begin{array}{lr}\text { yl)-2-thiophene Carboxamide (9b) S29-S30 } & \text { Sb }\end{array}$

${ }^{1} \mathrm{H}$ - and ${ }^{13} \mathrm{C}-\mathrm{NMR}$ spectra for 3-(4,4,5,5-Tetramethyl-1,3,2-dioxaborolan-2-yl)-3-

$\begin{array}{ll}\text { trimethylsilylthiophene }(\mathbf{9 c}) & \mathrm{S} 31-\mathrm{S} 32\end{array}$

${ }^{1} \mathrm{H}$ - and ${ }^{13} \mathrm{C}-\mathrm{NMR}$ spectra for 2-Nitro-3-(4,4,5,5-tetramethyl-1,3,2-dioxaborolan-2-yl)-

$\begin{array}{ll}\text { thiophene (9d) } & \text { S33-S34 }\end{array}$

${ }^{1} \mathrm{H}$ - and ${ }^{13} \mathrm{C}$-NMR spectra for 3-Acetyl-2-thienylboronic Acid (10a) S35-S36

${ }^{1} \mathrm{H}$ - and ${ }^{13} \mathrm{C}-\mathrm{NMR}$ spectra for 2-(4,4,5,5-Tetramethyl-1,3,2-dioxaborolan-2-yl)-3-

$\begin{array}{ll}\text { thienylcarbaldehyde (10c) } & \text { S37-S38 }\end{array}$

${ }^{1} \mathrm{H}$ - and ${ }^{13} \mathrm{C}-\mathrm{NMR}$ spectra for 3-Carboxyethyl-2-thiopheneboronic Acid (10d) S39-S40

${ }^{1} \mathrm{H}$ - and ${ }^{13} \mathrm{C}-\mathrm{NMR}$ spectra for $N$-tert-Butyl 3-(4-( $N^{\prime}, N^{\prime}$-dimethylamino $)$ phenyl)-2-

thiophene Carboxamide (11)

S41-S42 


\section{Experimental Section}

General Experimental Procedure: All reactions were performed in flame-dried equipment under nitrogen and magnetic stirring. Anhydrous THF, DMF, $\left(\mathrm{CH}_{3}\right)_{3} \mathrm{SiCl}$ and pinacolborane were purchased in Sure/Seal bottles. All other solvents were dried over molecular sieves (3A). Ethylmagnesium chloride in THF was purchased in Sure/Seal bottles and titrated using the procedure described by Lin and Paquette. ${ }^{1}$ Commercially available reagents and catalysts were used without further purification. Tri-tertbutylphosphine was purchased in ampoules and transferred to vials equipped with a septum under $\mathrm{N}_{2}$. The vial was stored at ambient temperature and gently heated $\left(\mathrm{P}(t-\mathrm{Bu})_{3}\right.$ is a gel at ambient temperature) with a heat gun prior to taking out aliquots with a syringe. Z-HSM-5 zeolite was purchased on $\mathrm{H}^{+}$-form from Zeolyst Inc. 2-Bromo-3-iodothiophene was prepared by bromination of 3-iodothiophene as described by Gronowitz et al. ${ }^{2}$ and 3-bromo-2nitrothiophene was prepared as described by Bacon et al. ${ }^{3}$ HPLC analyses were performed using a YMC $5 \mu \mathrm{m} 120 \AA ̊$ C-18 reversed phase column. A gradient system using acetonitrile and water (pH 2.3) over 35 min was used. UV-detection at $226 \mathrm{~nm}$ was applied. ${ }^{1} \mathrm{H}-\mathrm{NMR}$ and

${ }^{13} \mathrm{C}$-NMR spectra were recorded at $400 \mathrm{MHz}$ and $100 \mathrm{MHz}$, respectively. Mass spectra were recorded in the EI mode. Melting points were obtained on a differential scanning calorimeter. Melting intervals were determined as onset and endset temperatures.

3-Iodothiophene. A mixture of 2-iodothiophene (157.4 g, $0.75 \mathrm{~mol})$ and H-ZSM-5 zeolite (7.86 g) was stirred for 3 hours at $100^{\circ} \mathrm{C}$. After cooling to $60^{\circ} \mathrm{C}$ acetic acid $(160 \mathrm{~mL})$ and water $(80 \mathrm{~mL})$ were added. Zinc dust $(24.5 \mathrm{~g}, 0.375 \mathrm{~mol},<10 \mu \mathrm{m})$ was added under vigorous stirring in small portions at such a rate that the temperature was kept below $40^{\circ} \mathrm{C}$. After addition of the $\mathrm{Zn}$-dust the mixture was stirred at ambient temperature for further 16 hours. Water $(100 \mathrm{~mL})$ was added to the reaction mixture followed by extraction with MTBE $(3 \mathrm{x}$ $250 \mathrm{~mL}$ ). The combined organic phases were concentrated to approx. $300 \mathrm{~mL}$ under reduced 
pressure and washed with aq. $\mathrm{NaHCO}_{3}(500 \mathrm{~mL})$. The aqueous phase was extracted with MTBE $(100 \mathrm{~mL})$ and the combined organic phases were dried over $\mathrm{MgSO}_{4}$. The solvent was removed under reduced pressure. Distillation of the remaining oil afforded 3-iodothiophene (93.4 g, 59\%) as a colorless oil. bp $46-49^{\circ} \mathrm{C}$ (6 Torr). ${ }^{1} \mathrm{H}$ NMR data were in accordance with literature data. ${ }^{4}$

Typical Procedure for the Preparation of 3-Bromo-2-thienylmagnesium Chloride (2). A solution of 2,3-dibromothiophene (1) $(8.46 \mathrm{~g}, 35 \mathrm{mmol})$ in THF (30 mL) was cooled to $15^{\circ} \mathrm{C}$ under magnetic stirring. Ethylmagnesium chloride in $\mathrm{THF}(19.0 \mathrm{~mL}, 38 \mathrm{mmol})$ was added over $15 \mathrm{~min}$. The solution was stirred for further $15 \mathrm{~min}$ at $15^{\circ} \mathrm{C}$.

Typical procedure for the Preparation of 2-Bromo-3-thienylmagnesium Chloride (5). A solution of 2-bromo-3-iodothiophene (4) $(31.8 \mathrm{~g}, 0.11 \mathrm{~mol})$ in THF (50 mL) was cooled to $15^{\circ} \mathrm{C}$ under magnetic stirring. Ethylmagnesium chloride in $\mathrm{THF}(58.5 \mathrm{~mL}, 0.112 \mathrm{~mol})$ was added over $15 \mathrm{~min}$. The solution was stirred for further $15 \mathrm{~min}$ at $15^{\circ} \mathrm{C}$.

Ethyl 3-Bromo-2-thiophenecarboxylate (7a). A THF-solution of 2 (63.3 mmol) cooled to $15^{\circ} \mathrm{C}$ was added to ethyl cyanoformate $(6.54 \mathrm{~mL}, 66 \mathrm{mmol})$ in $\mathrm{THF}(20 \mathrm{~mL})$ at $25-30^{\circ} \mathrm{C}$ over $30 \mathrm{~min}$. The reaction mixture was stirred for further $30 \mathrm{~min}$ at $20-25^{\circ} \mathrm{C}$ followed by addition of sat. $\mathrm{NaHCO}_{3}(100 \mathrm{~mL})$. The mixture was vigorously stirred until all solid material had dissolved and the solution was extracted with MTBE (2 x $100 \mathrm{~mL})$. The combined organic phases were dried over $\mathrm{MgSO}_{4}$ and the solvents were removed under reduced pressure. The resulting brown oil was distilled on a $30 \mathrm{~cm}$ Vigreux column affording 7a as a colorless oil (8.4 g, 59\%): bp $77-80^{\circ} \mathrm{C}\left(0.8\right.$ Torr) (lit. ${ }^{5} 63-66^{\circ} \mathrm{C}(0.8$ Torr $\left.)\right) ;{ }^{1} \mathrm{H}-\mathrm{NMR}\left(\mathrm{CDCl}_{3}\right)^{6} \delta 1.39(\mathrm{t}, J=$ $7.3 \mathrm{~Hz}, 3 \mathrm{H}), 4.37$ (q, $J=7.3 \mathrm{~Hz}, 2 \mathrm{H}), 7.08(\mathrm{~d}, J=5.3 \mathrm{~Hz}, 1 \mathrm{H}), 7.45(\mathrm{~d}, J=5.3 \mathrm{~Hz}, 1 \mathrm{H})$;

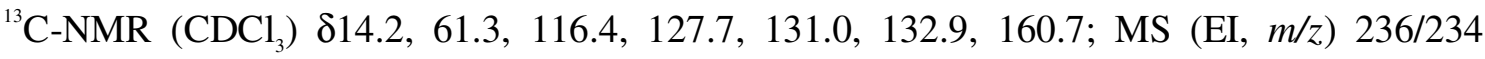
(29/30), 208/206 (40/41), 191/189 (99/100), 82 (62), 45 (49). 
$N$-tert-Butyl 3-Bromothienyl-2-carboxamide (7b). To a THF-solution of 2 (35 mmol) was added tert-butyl isocyanate $(8.0 \mathrm{~mL}, 70 \mathrm{mmol})$ at $25-30^{\circ} \mathrm{C}$ over $15 \mathrm{~min}$. The reaction mixture was stirred for further $45 \mathrm{~min}$ at $20-25^{\circ} \mathrm{C}$ followed by addition of $1 \mathrm{M} \mathrm{HCl}(50 \mathrm{~mL})$. The mixture was extracted with MTBE $(2 \times 50 \mathrm{~mL})$. The combined organic phases were washed with water $(50 \mathrm{~mL})$ and dried over $\mathrm{MgSO}_{4}$. The solvents were removed under reduced pressure and the crude product was re-crystallized from heptane to give $\mathbf{7 b}$ as white needles (6.98 g, 76\%): mp 72.2-76.0 ${ }^{\circ}$; ${ }^{1} \mathrm{H}-\mathrm{NMR}\left(\mathrm{CDCl}_{3}\right) \delta 1.47$ (s, $\left.9 \mathrm{H}\right), 6.90-6.98$ (broad s, $1 \mathrm{H}$ ), $7.00(\mathrm{~d}, J=5.3 \mathrm{~Hz}, 1 \mathrm{H}), 7.40(\mathrm{~d}, J=5.3 \mathrm{~Hz}, 1 \mathrm{H}) ;{ }^{13} \mathrm{C}-\mathrm{NMR}\left(\mathrm{CDCl}_{3}\right) \delta 28.9,52.3,107.6$, 129.4, 131.7, 136.5, 159.3; MS (EI, m/z) 263/261 (9/8), 248/246 (25/24), 191/189 (100/99), 182 (10), 163/161 (3/3), 82 (29). Anal. Calcd. for $\mathrm{C}_{9} \mathrm{H}_{12}$ BrNOS C 41.23, H 4.61, N 5.34, Br 30.48, found C 41.20, H 4.60, N 5.30, Br 30.67.

3-Bromo-2-trimethylsilylthiophene (7c). To a THF-solution of 2 (25 mmol) was added trimethylsilyl chloride $(3.5 \mathrm{~mL}, 28 \mathrm{mmol})$ at $20-25^{\circ} \mathrm{C}$ over $5 \mathrm{~min}$. The reaction mixture was stirred for 3 hours at $22-25^{\circ} \mathrm{C}$ followed by addition of sat. $\mathrm{NH}_{4} \mathrm{Cl}(50 \mathrm{~mL})$. The mixture was extracted with heptane $(2 \times 25 \mathrm{~mL})$. The combined organic phases were dried over $\mathrm{MgSO}_{4}$ and the solvents were removed under reduced pressure. Kugelrohr distillation gave 7c as a colorless oil (4.8 g, 81\%): bp $100-103^{\circ} \mathrm{C}$ (1 Torr) (lit. ${ }^{7} 110^{\circ} \mathrm{C}\left(2\right.$ Torr)). ${ }^{1} \mathrm{H}$ NMR and ${ }^{13} \mathrm{C}$ NMR data were in accordance with published data. ${ }^{8}$

3-Acetyl-2-bromothiophene (8a). ${ }^{9} \quad$ A THF-solution of $5(0.10 \mathrm{~mol})$ cooled to $15^{\circ} \mathrm{C}$ was added to acetic anhydride $(14.2 \mathrm{~mL}, 0.15 \mathrm{~mol})$ in THF $(40 \mathrm{~mL})$ at $25-30^{\circ} \mathrm{C}$ over $30 \mathrm{~min}$. The reaction mixture was stirred for further $45 \mathrm{~min}$ at $20-25^{\circ} \mathrm{C}$ followed by addition of $1 \mathrm{M} \mathrm{HCl}$ $(150 \mathrm{~mL})$. The mixture was extracted with $\operatorname{MTBE}(2 \times 50 \mathrm{~mL})$. The combined organic phases were washed with $4 \mathrm{M} \mathrm{KOH}(100 \mathrm{~mL})$, water $(50 \mathrm{~mL})$ and dried over $\mathrm{MgSO}_{4}$. The solvents were removed under reduced pressure. Distillation on a $30 \mathrm{~cm}$ Vigreux column afforded 8a as 
a slight yellow oil (14.2 g, 69\%; purity: > 95\% (HPLC)): bp 56-61 ${ }^{\circ} \mathrm{C}$ (0.4 Torr).

Crystallization from heptane gave a product containing approx. 3\% of 2-acetyl-3bromothiophene: ${ }^{10} \mathrm{mp} 32.7-36.7^{\circ} \mathrm{C} ;{ }^{1} \mathrm{H}-\mathrm{NMR}\left(\mathrm{CDCl}_{3}\right) \delta 2.60(\mathrm{~s}, 3 \mathrm{H}), 7.21(\mathrm{~d}, J=5.8 \mathrm{~Hz}, 1$ H), $7.33(\mathrm{~d}, J=5.8 \mathrm{~Hz}, 1 \mathrm{H}) ;{ }^{13} \mathrm{C}-\mathrm{NMR}\left(\mathrm{CDCl}_{3}\right) \delta 29.9,117.9,126.0,129.0,139.3,192.1$; MS( EI, m/z): 206/204 (34/32), 191/189 (100/95), 163/161 (8/6), 82, (43), 45 (43). Anal. Calcd. for $\mathrm{C}_{6} \mathrm{H}_{5} \mathrm{BrOS} \mathrm{C} 35.14, \mathrm{H} 2.46, \mathrm{Br} 38.96$, found C 35.00, H 2.40, Br 38.81 .

2-Bromo-3-cyanothiophene (8b). A THF-solution of $5(0.11 \mathrm{~mol})$ cooled to $1{ }^{\circ} \mathrm{C}$ was added to $p$-toluenesulfonyl cyanide $(20.3 \mathrm{~g}, 0.11 \mathrm{~mol})$ in $\mathrm{THF}(100 \mathrm{~mL})$ at $2-7^{\circ} \mathrm{C}$ over $30 \mathrm{~min}$. The reaction mixture was stirred for further 1 hour at $20-25^{\circ} \mathrm{C}$ followed by addition of sat. $\mathrm{NaHCO}_{3}(600 \mathrm{~mL})$ and MTBE $(100 \mathrm{~mL})$. The mixture was extracted with $\mathrm{CH}_{2} \mathrm{Cl}_{2}(3 \times 100$ $\mathrm{mL}$ ) and the combined organic phases were dried over $\mathrm{MgSO}_{4}$. The solvents were removed under reduced pressure. Distillation on a $30 \mathrm{~cm}$ Vigreux column afforded $\mathbf{8 b}$ as a colorless oil (11.5 g, 64\%; purity: $\left.\approx 90 \%\left({ }^{1} \mathrm{H}-\mathrm{NMR}\right)\right)$; bp $82-85^{\circ} \mathrm{C}$ (3 Torr) (lit. ${ }^{11}$ bp $130^{\circ} \mathrm{C}$ (22 Torr)). Crystallization of the oil from heptane gave a product containing 4\% of 3-bromo-2cyanothiophene: ${ }^{12} \mathrm{mp} 20.2-27.4^{\circ} \mathrm{C} ;{ }^{1} \mathrm{H}-\mathrm{NMR}\left(\mathrm{CDCl}_{3}\right) \delta 7.12(\mathrm{~d}, J=5.6 \mathrm{~Hz}, 1 \mathrm{H}), 7.34(\mathrm{~d}, J=$ $5.6 \mathrm{~Hz}, 1 \mathrm{H}) ;{ }^{13} \mathrm{C}-\mathrm{NMR}\left(\mathrm{CDCl}_{3}\right) \delta 113.7,114.0,123.4,128.1,129.0$; MS (EI, $\left.m / z\right)$ 189/187 (100), 108 (73), 82 (37), 64 (91), 45 (85). Anal. Calcd. for $\mathrm{C}_{5} \mathrm{H}_{2} \mathrm{BrNS}$ C 31.94, H 1.07, N 7.45, found C 31.91, H 1.04, N 7.66.

2-Bromo-3-thienylcarbaldehyde $(\mathbf{8 c})$. To a cooled $\left(11^{\circ} \mathrm{C}\right)$ THF-solution of 5 (35 mmol) was added DMF $(5.4 \mathrm{~mL}, 70 \mathrm{mmol})$ at $20-30^{\circ} \mathrm{C}$ over $5 \mathrm{~min}$. The reaction mixture was stirred for further 1 hour at $20-25^{\circ} \mathrm{C}$ followed by addition of $1 \mathrm{M} \mathrm{HCl}(100 \mathrm{~mL})$. The mixture was extracted with MTBE ( $2 \times 50 \mathrm{~mL})$. The combined organic phases were washed with sat. $\mathrm{NaHCO}_{3}(50 \mathrm{~mL})$ and dried over $\mathrm{MgSO}_{4}$. The solvents were removed under reduced pressure resulting in a brown oil. The oil was dissolved in heptane $(50 \mathrm{~mL})$ and kept at $2-4^{\circ} \mathrm{C}$ for 3 
weeks which gave 8c as pale yellow needless (3.2 g, 48\%; purity: 97\% (GC)): mp 28.5$34.2^{\circ} \mathrm{C}$ (lit. ${ }^{11} \mathrm{mp} 34-35^{\circ} \mathrm{C}$ ). Further crystallization from heptane gave the analytically pure product: $\mathrm{mp} 34.1-39.3^{\circ} \mathrm{C} ;{ }^{1} \mathrm{H}-\mathrm{NMR}\left(\mathrm{CDCl}_{3}\right)^{32} \delta 7.28(\mathrm{dd}, J=5.9,0.7 \mathrm{~Hz}, 1 \mathrm{H}), 7.36(\mathrm{~d}, \mathrm{~J}=5.9$ $\mathrm{Hz}, 1 \mathrm{H}), 9.94(\mathrm{~d}, J=0.7 \mathrm{~Hz}, 1 \mathrm{H}) ;{ }^{13} \mathrm{C}-\mathrm{NMR}\left(\mathrm{CDCl}_{3}\right) \delta 125.2,126.2,126.9,138.5,184.6$; MS (EI, m/z) 192/190 (73/73), 191/189 (100/99), 163/161 (9/8), 119/117 (6/6), 82 (51), 57 (23), 45 (47).

Ethyl 2-Bromo-3-thiophenecarboxylate (8d). A THF-solution of 5 (40 mmol) cooled to $1{ }^{\circ} \mathrm{C}$ was added to ethyl cyanoformate $(4.4 \mathrm{~mL}, 44 \mathrm{mmol})$ in $\mathrm{THF}(20 \mathrm{~mL})$ at $4-7^{\circ} \mathrm{C}$ over 20 min. The reaction mixture was stirred for further $30 \mathrm{~min}$ at $20-25^{\circ} \mathrm{C}$ followed by addition of sat. $\mathrm{NaHCO}_{3}(100 \mathrm{~mL})$. The mixture was extracted with MTBE $(2 \mathrm{x} 100 \mathrm{~mL})$ and the combined organic phases were dried over $\mathrm{MgSO}_{4}$. The solvents were removed under reduced pressure. Distillation on Podbielniak column afforded 8d as a colorless oil (4.6 g, 49\%) (The product contained $4 \%$ of $7 \mathbf{a})$ : bp $79-81^{\circ} \mathrm{C}(0.7$ Torr $) ;{ }^{1} \mathrm{H}-\mathrm{NMR}\left(\mathrm{CDCl}_{3}\right) \delta 1.39(\mathrm{t}, J=7.3 \mathrm{~Hz}, 3$ $\mathrm{H}), 4.35(\mathrm{q}, J=7.3 \mathrm{~Hz}, 2 \mathrm{H}), 7.21(\mathrm{~d}, J=5.8 \mathrm{~Hz}, 1 \mathrm{H}), 7.36(\mathrm{~d}, J=5.8 \mathrm{~Hz}, 1 \mathrm{H}) ;{ }^{13} \mathrm{C}-\mathrm{NMR}$ $\left(\mathrm{CDCl}_{3}\right) \delta 14.2,60.9,119.6,125.7,129.3,131.3,161.9 ; \mathrm{MS}(\mathrm{EI}, \mathrm{m} / \mathrm{z})$ 236/234 (25/23), 208/206 (33/31), 191/189 (100/99), 82 (74), 45 (60). Anal. Calcd. for $\mathrm{C}_{7} \mathrm{H}_{7} \mathrm{BrO}_{2} \mathrm{~S} \mathrm{C} 35.76, \mathrm{H}$ 3.00, found C 35.30, H 3.10 .

General Procedure for the Borylation of 2,3-Substituted Bromothiophenes with Pinacolborane Catalyzed by $\mathbf{P d}\left(\mathbf{P}(t-\mathrm{Bu})_{3}\right)_{2}$. The specified amount of $\operatorname{Pd}\left(\mathrm{P}(t-\mathrm{Bu})_{3}\right)_{2}$ (weighed in the air) was added to 2,3-substituted bromothiophenes 7a-d or 8a-d (1 eqv.) dissolved in dry THF ( $2 \mathrm{ml} / \mathrm{mmol})$. Triethylamine (3 eqv.) and pinacolborane (1.5 eqv.) were added to the mixture. The reaction mixture was stirred at $40^{\circ} \mathrm{C}$ under $\mathrm{N}_{2}$ for the specified time followed by cooling to room temperature and filtration. The solid was extracted with MTBE and the filtrates were combined. The solvents were removed under reduced pressure. 
2-Carboxyethyl-3-thienylboronic Acid (9a). This was prepared from 7a (4.70 g, 20 $\mathrm{mmol})$. The evaporation residue was re-dissolved in THF $(20 \mathrm{~mL})$ and water $(20 \mathrm{~mL})$. The mixture was adjusted to $\mathrm{pH} 3.5$ by acetic acid and stirred vigorously for $50 \mathrm{~min}$, this resulted in precipitation of a light brown solid. The mixture was filtered and the solid was washed with heptane $(2 \times 5 \mathrm{~mL})$ and cold water $(2 \times 5 \mathrm{~mL})$ giving 9a $(3.51 \mathrm{~g}, 88 \%$; purity: $\approx 95 \%$ (HPLC)). Re-crystallization from toluene gave the pure product: mp $138.3-150.7^{\circ} \mathrm{C}$; ${ }^{1} \mathrm{H}-\mathrm{NMR}$ (DMSO$\left.\mathrm{d}_{6}\right) \delta 1.34(\mathrm{t}, J=6.8 \mathrm{~Hz}, 3 \mathrm{H}), 4.34(\mathrm{q}, J=6.8 \mathrm{~Hz}, 2 \mathrm{H}), 7.34(\mathrm{~d}, J=5.0 \mathrm{~Hz}, 1 \mathrm{H}), 7.89(\mathrm{~d}, J=$ $5.0 \mathrm{~Hz}, 1 \mathrm{H}), 8.48(\mathrm{~s}, 2 \mathrm{H}) ;{ }^{13} \mathrm{C}-\mathrm{NMR}\left(\mathrm{DMSO}_{6}\right) \delta 14.2,61.6,132.6,133.9,136.0,163.8$. Anal. Calcd. for $\mathrm{C}_{7} \mathrm{H}_{9} \mathrm{BO}_{4} \mathrm{~S} \mathrm{C} 42.03, \mathrm{H} 4.54$, found $\mathrm{C} 42.25, \mathrm{H} 4.53$.

\section{$N$-tert-Butyl 3-(4,4,5,5-tetramethyl-1,3,2-dioxaborolan-2-yl)-2-thiophene Carboxamide}

(9b). This was prepared from $7 \mathbf{b}(787 \mathrm{mg}, 3 \mathrm{mmol})$. The evaporation residue was re-dissolved in heptane $(5 \mathrm{~mL})$ and washed with water $(3 \times 10 \mathrm{~mL})$ adjusted to $\mathrm{pH} 4$. The combined organic phases were dried over $\mathrm{MgSO}_{4}$ and the solvent was removed under reduced pressure. The brown oil was distilled at $130-138^{\circ} \mathrm{C}(0.5$ Torr $)$ and $9 \mathbf{b}(505 \mathrm{mg}, 54 \%)$ was collected as white crystals by condensation on a water cooled "cold finger": mp 100.6-105.0 ${ }^{\circ} \mathrm{C} ;{ }^{1} \mathrm{H}-\mathrm{NMR}$ $\left(\mathrm{CDCl}_{3}\right) \delta 1.38(\mathrm{~s}, 12 \mathrm{H}), 1.48(\mathrm{~s}, 9 \mathrm{H}) 7.38(\mathrm{~d}, J=5.7 \mathrm{~Hz}, 1 \mathrm{H}), 7.42(\mathrm{~d}, J=5.7 \mathrm{~Hz}, 1 \mathrm{H}), 8.6-$ 8.68 (broad s, $1 \mathrm{H}) ;{ }^{13} \mathrm{C}-\mathrm{NMR}\left(\mathrm{CDCl}_{3}\right) \delta 24.8,28.8,51.9,84.9,128.4,135.86,153.4,161.5$. MS (EI, m/z): 309 (5), 294 (5), 276 (3), 251 (29), 237 (18), 195 (56), 154 (38), 137 (54), 83 (100), 59 (25). Anal. Calcd. for $\mathrm{C}_{15} \mathrm{H}_{24} \mathrm{BNO}_{3} \mathrm{~S} \mathrm{C} 58.26, \mathrm{H}$ 7.82, N 4.53, found C 58.17, H 7.94, N 4.48 .

3-(4,4,5,5-Tetramethyl-1,3,2-dioxaborolan-2-yl)-3-trimethylsilylthiophene (9c). This was prepared from 7c $(247 \mathrm{mg}, 1.05 \mathrm{mmol})$. The evaporation residue was re-dissolved in $\mathrm{CH}_{2} \mathrm{Cl}_{2}$ and subjected to column chromatography on silica gel (heptane/ $\mathrm{CH}_{2} \mathrm{Cl}_{2}(1: 1), \mathrm{R}_{\mathrm{f}}=0.68$ ) to give 9c (171 mg, 58\%): $\mathrm{mp} 64.4-68.5^{\circ} \mathrm{C} ;{ }^{1} \mathrm{H}-\mathrm{NMR}\left(\mathrm{CDCl}_{3}\right) \delta 0.39(\mathrm{~s}, 9 \mathrm{H}), 1.33(\mathrm{~s}, 12 \mathrm{H})$, 
$7.52(\mathrm{~d}, J=4.6 \mathrm{~Hz}, 1 \mathrm{H}), 7.59(\mathrm{~d}, J=4.6 \mathrm{~Hz}, 1 \mathrm{H}) ;{ }^{13} \mathrm{C}-\mathrm{NMR}\left(\mathrm{CDCl}_{3}\right) \delta$ 0.2, 24.8, 83.5, 128.8, 136.1, 152.6; MS(EI, m/z) 282 (12), 267 (40), 185 (36), 167 (15), 125 (13), 83 (100), 55 (40). Anal. Calcd. for $\mathrm{C}_{13} \mathrm{H}_{23} \mathrm{SiBO}_{2} \mathrm{~S} \mathrm{C} 55.31, \mathrm{H}$ 8.21, found C 55.46, H 8.32.

2-Nitro-3-(4,4,5,5-tetramethyl-1,3,2-dioxaborolan-2-yl)-thiophene (9d). This was prepared from $7 \mathbf{d}(625 \mathrm{mg}, 3 \mathrm{mmol})$. The evaporation residue was distilled at $95-105^{\circ} \mathrm{C}(0.3$ Torr) and 9d (329 mg, 43\%) was collected as pale orange crystals by condensation on a water cooled "cold finger": mp 80.9-84.0 ${ }^{\circ} \mathrm{C} ;{ }^{1} \mathrm{H}-\mathrm{NMR}\left(\mathrm{CDCl}_{3}\right) \delta 1.41(\mathrm{~s}, 12 \mathrm{H}), 7.08(\mathrm{~d}, J=5.2 \mathrm{~Hz}$, $1 \mathrm{H}), 7.52(\mathrm{~d}, J=5.2 \mathrm{~Hz}, 1 \mathrm{H}) ;{ }^{13} \mathrm{C}-\mathrm{NMR}\left(\mathrm{CDCl}_{3}\right) \delta 24.7,85.1,130.7,132.3 ; \mathrm{MS}(\mathrm{EI}, \mathrm{m} / z)$ 255 (<5), 240 (11), 154 (56), 139 (100). Anal. Calcd. for $\mathrm{C}_{10} \mathrm{H}_{14} \mathrm{BNO}_{4} \mathrm{~S} \mathrm{C} 47.08, \mathrm{H}$ 5.53, N 5.49, found C 47.20, H 5.57, N 5.43.

3-Acetyl-2-thienylboronic Acid (10a). ${ }^{13}$ This was prepared from 8 a $(2.99 \mathrm{~g}, 14.6 \mathrm{mmol})$. The evaporation residue was re-dissolved in THF $(5 \mathrm{~mL})$ and water $(3 \mathrm{~mL})$. The mixture was adjusted to $\mathrm{pH} 4$ by acetic acid and stirred vigorously for 45 min resulting in precipitation of a blue-green solid. The mixture was filtered and the solid was washed with heptane $(2 \mathrm{x} 10 \mathrm{~mL})$ and cold water $(4 \mathrm{~mL})$ resulting in $10 a\left(527 \mathrm{mg}, 24 \%\right.$; purity: $\approx 95 \%$ ( $\left.\left.{ }^{1} \mathrm{H}-\mathrm{NMR}\right)\right)$. Recrystallization from toluene gave the pure product: $\mathrm{mp}$ dec. $\approx 125^{\circ} \mathrm{C}\left(\right.$ lit. $^{13}$ dec. $\left.120-140^{\circ} \mathrm{C}\right)$; ${ }^{1} \mathrm{H}-\mathrm{NMR}\left(\mathrm{DMSO}_{6}\right)^{13} \delta 2.68(\mathrm{~s}, 3 \mathrm{H}), 7.84(\mathrm{~d}, J=5.2 \mathrm{~Hz}, 1 \mathrm{H}), 7.89(\mathrm{~d}, J=5.2 \mathrm{~Hz}, 1 \mathrm{H})$, $9.06(\mathrm{~s}, 2 \mathrm{H}) ;{ }^{13} \mathrm{C}-\mathrm{NMR}\left(\mathrm{DMSO}-\mathrm{d}_{6}\right) \delta 229.5,131.0,131.9,145.3,197.9$.

2-(4,4,5,5-Tetramethyl-1,3,2-dioxaborolan-2-yl)-3-thienylcarbaldehyde (10c). This was prepared from $8 \mathbf{c}(1.91 \mathrm{~g}, 10 \mathrm{mmol})$. The evaporation residue was re-dissolved in heptane (20 $\mathrm{mL})$ and washed with water $(3 \times 10 \mathrm{~mL})$ adjusted to $\mathrm{pH} 4$. The combined organic phases were dried over $\mathrm{MgSO}_{4}$ and the solvent was removed under reduced pressure. Re-crystallization from heptane gave 10c $(1.17 \mathrm{~g}, 49 \%$; purity: $\approx 93 \%$ (HPLC)). A further re-crystallization from ethyl acetate gave the pure product: $\mathrm{mp} 65.3-68.3^{\circ} \mathrm{C} ;{ }^{1} \mathrm{H}-\mathrm{NMR}\left(\mathrm{CDCl}_{3}\right) \delta 1.38(\mathrm{~s}, 12 \mathrm{H})$, 
$7.57(\mathrm{~d}, J=4.9 \mathrm{~Hz}, 1 \mathrm{H}), 7.68(\mathrm{~d}, J=4.9 \mathrm{~Hz}, 1 \mathrm{H}), 10.41(\mathrm{~s}, 1 \mathrm{H}) ;{ }^{13} \mathrm{C}-\mathrm{NMR}\left(\mathrm{CDCl}_{3}\right) \delta 24.8$, 84.8, 127.0, 132.3, 150.2, 187.9; MS(EI, m/z) 238 (1), 223 (5), 182 (81), 180 (100), 165 (28), 139 (70), 111 (23), 84 (60), 57 (55). Anal. Calcd. for $\mathrm{C}_{11} \mathrm{H}_{15} \mathrm{BO}_{3} \mathrm{~S} \mathrm{C} 55.49$, H 6.35, found: $\mathrm{C}$ 55.40, H 6.30 .

3-Carboxyethyl-2-thiopheneboronic Acid (10d). This was prepared form 8d (2.82 g, 12 $\mathrm{mmol})$. The evaporation residue was re-dissolved in THF $(10 \mathrm{~mL})$ and water $(10 \mathrm{~mL})$. The mixture was adjusted to $\mathrm{pH} 4$ by acetic acid and vigorously stirred for 25 min resulting in precipitation of a light brown solid. The slurry was filtered and the solid was washed with heptane $(2 \times 10 \mathrm{~mL})$ and cold water $(2 \times 10 \mathrm{~mL})$ resulting in $10 d(1.53 \mathrm{~g}, 64 \%$; purity: $\approx 95 \%$ (NMR)). Re-crystallization from toluene gave the title compound containing 5\% of 9a. The product was purified by preparative HPLC: $\mathrm{mp} 124.4-134.6^{\circ} \mathrm{C}$. Dec. $\approx 156^{\circ} \mathrm{C} ;{ }^{1} \mathrm{H}-\mathrm{NMR}$ $\left(\mathrm{DMSO}_{6}\right) \delta 1.35(\mathrm{t}, J=6.6 \mathrm{~Hz}, 3 \mathrm{H}), 4.35(\mathrm{q}, J=6.6 \mathrm{~Hz}, 2 \mathrm{H}), 7.59(\mathrm{~d}, J=4.6 \mathrm{~Hz}, 1 \mathrm{H})$, $7.82(\mathrm{~d}, J=4.6 \mathrm{~Hz}, 1 \mathrm{H}), 8.86(\mathrm{~s}, 2 \mathrm{H}) ;{ }^{13} \mathrm{C}-\mathrm{NMR}\left(\mathrm{DMSO}_{\mathrm{d}}\right) \delta$ 14.9, 62.3, 131.0, 131.7, 137.6, 166.2. Anal Calcd. for $\mathrm{C}_{7} \mathrm{H}_{9} \mathrm{BO}_{4} \mathrm{~S} \mathrm{C} 42.03, \mathrm{H} 4.54$, found: $\mathrm{C} 42.01, \mathrm{H} 4.46$.

$N$-tert-Butyl 3-(4-(N’,N'-dimethylamino)phenyl)-2-thiophene Carboxamide (11). Crude 9b was prepared from $7 \mathbf{b}(262 \mathrm{mg}, 1.0 \mathrm{mmol})$ according to the general borylation procedure described above. The evaporation residue was dissolved in DMF (2 mL) and 4-bromo- $N, N$ dimethylaniline $(304 \mathrm{mg}, 1.5 \mathrm{mmol}), \mathrm{Pd}\left(\mathrm{P}(t-\mathrm{Bu})_{3}\right)_{2}(9.7 \mathrm{mg}, 0.019 \mathrm{mmol})$ and anhydrous $\mathrm{K}_{3} \mathrm{PO}_{4}$ (flame dried) $(636 \mathrm{mg}, 3.0 \mathrm{mmol})$ were added to the solution. The reaction mixture was stirred at $50^{\circ} \mathrm{C}$ for 18 hours and at $100^{\circ} \mathrm{C}$ for 2 hours. $1 \mathrm{M} \mathrm{HCl}(6 \mathrm{~mL})$ was added to the reaction mixture followed by extraction with MTBE $(6 \mathrm{~mL})$. The organic phase was extracted with $1 \mathrm{M} \mathrm{HCl}(2 \times 5 \mathrm{~mL})$. The combined aqueous phases were basified with $4 \mathrm{M} \mathrm{KOH}$ and extracted with MTBE (3 x $5 \mathrm{~mL})$. The solvent was removed under reduced pressure and the crude product was purified by flash chromatography on silica gel (ethyl acetate/heptane $(3: 7)$, $\left.\mathrm{R}_{\mathrm{f}}=0.33\right)$ affording $\mathbf{1 1}(179 \mathrm{mg}, 59 \%$ overall yield $)$ of white crystals: $\mathrm{mp} 151.1-154.8{ }^{\circ} \mathrm{C} ;{ }^{1} \mathrm{H}-$ 
NMR $\left(\mathrm{CDCl}_{3}\right) \delta 1.20(\mathrm{~s}, 9 \mathrm{H}), 3.00(\mathrm{~s}, 6 \mathrm{H}), 6.78(\mathrm{dt}, J=8.5,2.7 \mathrm{~Hz}, 2 \mathrm{H}), 6.95(\mathrm{~d}, J=5.1$

$\mathrm{Hz}, 1 \mathrm{H}), 7.27(\mathrm{dt}, J=8.5,2.7 \mathrm{~Hz}, 2 \mathrm{H}), 7.37(\mathrm{~d}, J=5.1 \mathrm{~Hz}, 1 \mathrm{H}) ;{ }^{13} \mathrm{C}-\mathrm{NMR}\left(\mathrm{CDCl}_{3}\right) \delta 28.6$, 40.4, 51.2, 112.4, 122.7, 127.7, 130.1, 130.8, 135.7, 141.8, 150.6, 161.8; MS(EI, m/z) 302 (100), 246 (49), 245 (48), 230 (92), 202 (14), 187 (14), 186 (14), 114 (43), 79 (27). Anal. Calcd. for $\mathrm{C}_{17} \mathrm{H}_{22} \mathrm{~N}_{2} \mathrm{OS} \mathrm{C}$ 67.51, H 7.33, N 9.26, found C 67.36, H 7.48, N 9.13.

\section{References}

(1) Lin, S.-H.; Paquette, L. A. Synth. Commun. 1994, 24, 2503-2506.

(2) Gronowitz, S.; Petterson, K. J. Heterocycl. Chem. 1976, 13, 1099-1100.

(3) Bacon, R. G. R.; Hamilton, S. D. J. Chem. Soc., Perkin Trans. 1 1974, 1970-1975.

(4) Satonaka, H. Bull. Chem. Soc. Jpn. 1983, 56, 2463-2468.

(5) Chadwick, D. J.; Chambers, J.; Meakins, G. D.; Snowden, R. L. J. Chem. Soc., Perkin Trans. 1 1973, 1766-1773.

(6) Gromova, G. P.; Belin`kii, L. I.; Krayushkin, M. M. Chem. Heterocycl. Compd. (Engl. Transl.) 1993, 29, 889-891.

(7) Seconi, G.; Eaborn, C.; Stamper, J. G. J. Organometal. Chem. 1981, 204, 153-168.

(8) Fröhlich, J.; Kalt, W. J. Org. Chem. 1990, 55, 2993-2995.

(9) The compound was indicated but no characterization data were presented in: Noto, R.; Frenna, V.; Consiglio, G.; Spinelli, D. J. Chem. Res., Miniprint 1991, 2701-2710.

(10) Muratake, H.; Hayakawa, A.; Natsume, M. Chem. Pharm. Bull. 2000, 48, 1558-1566.

(11) Fournai, P.; Guillard. R.; Person, M. Bull. Soc. Chim. Fr. 1967, 4115-4120. 
(12) Crude 3-bromo-2-cyanothiophene was prepared from 3-bromo-2-thienylmagnesium chloride and $p$-toluenesulfonyl cyanide according to the procedures described in the Experimental section. ${ }^{1} \mathrm{H}-\mathrm{NMR}$ data for this product: ${ }^{1} \mathrm{H}-\mathrm{NMR}\left(\mathrm{CDCl}_{3}\right) \delta 7.11(\mathrm{~d}, J=$ $4.9 \mathrm{~Hz}, 1 \mathrm{H}), 7.54(\mathrm{~d}, J=4.9 \mathrm{~Hz}, 1 \mathrm{H})$. These data are in accordance with the data observed for the by-product in $\mathbf{8 b}$.

(13) Gronowitz, S.; Dahlgreen, T.; Namtvedt, J.; Roos, C.; Rosén, G.; Sjöberg, B.; Forsgren, U. Acta Pharm. Suecica 1971, 8, 623-638. 


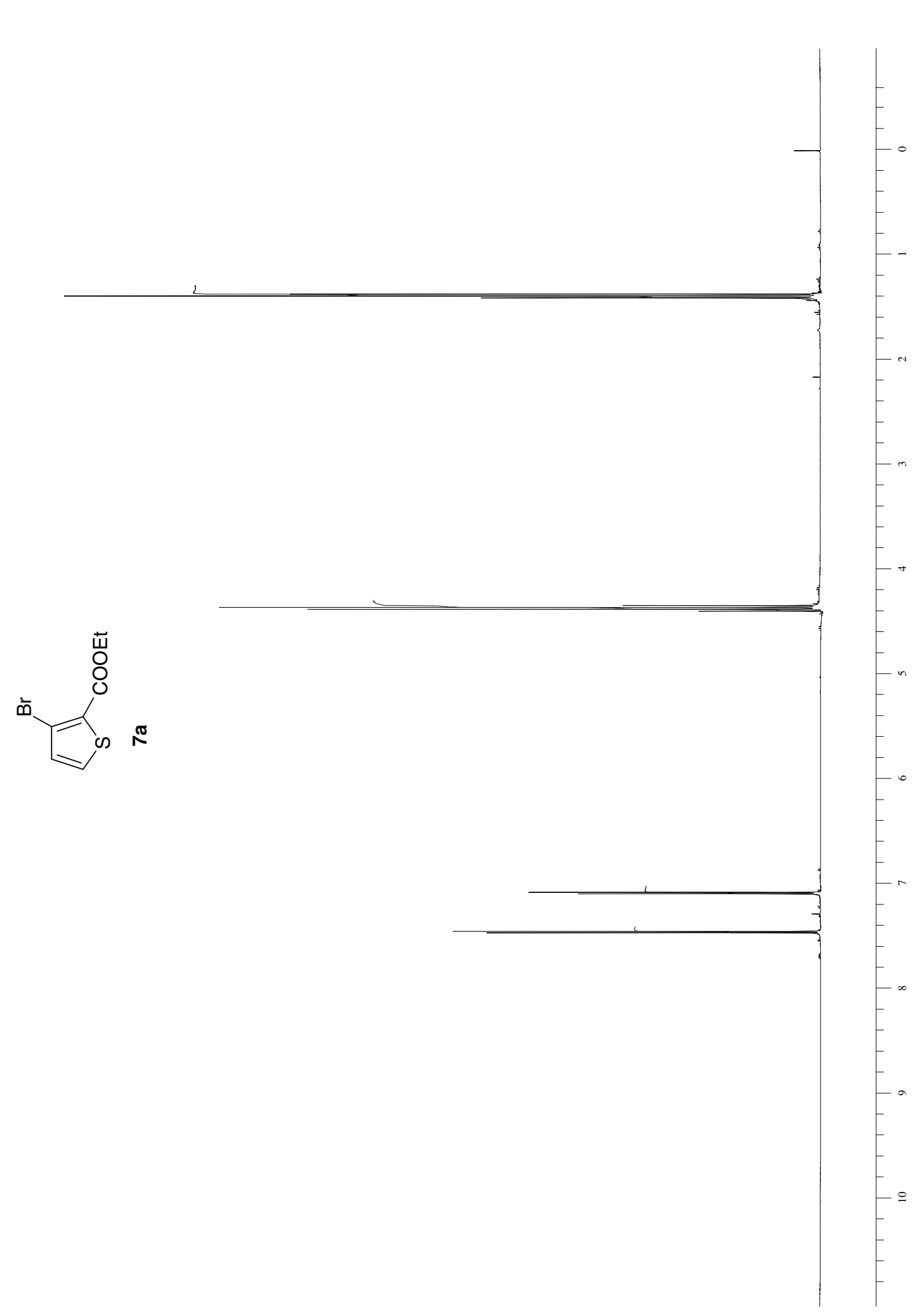

$\frac{n}{n}$ 


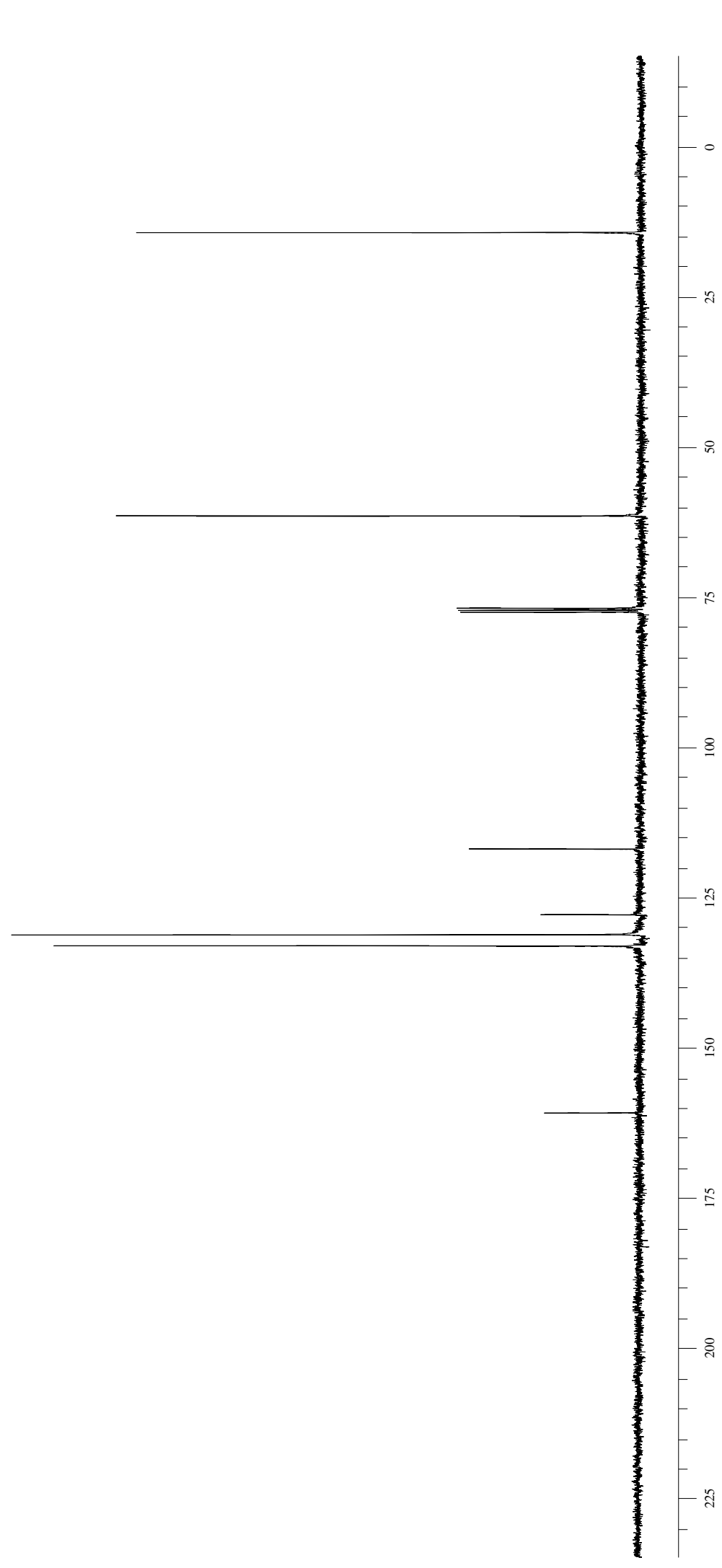

文

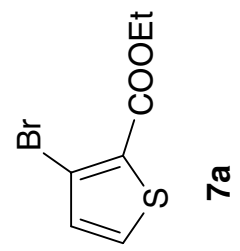




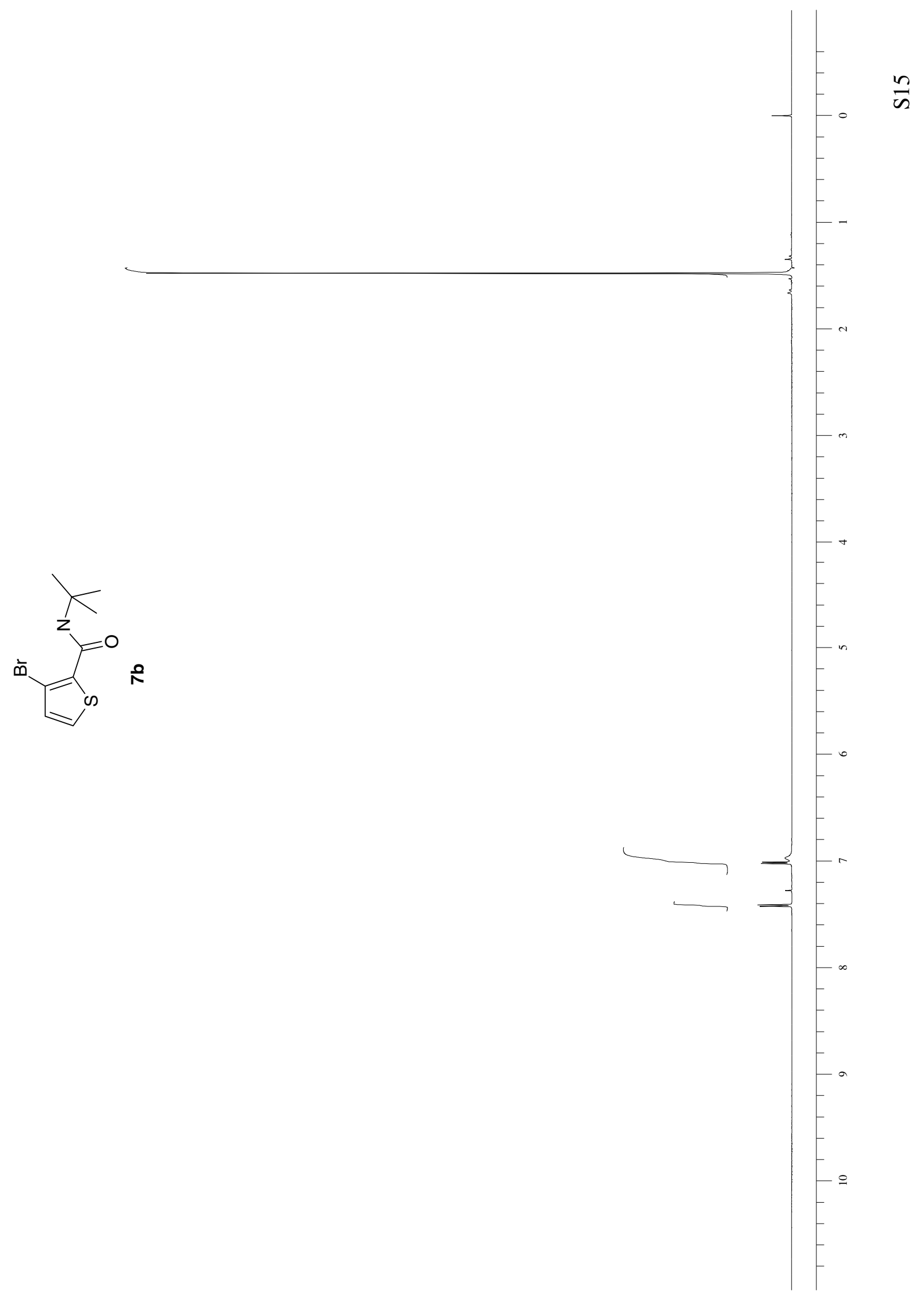




$$
1
$$




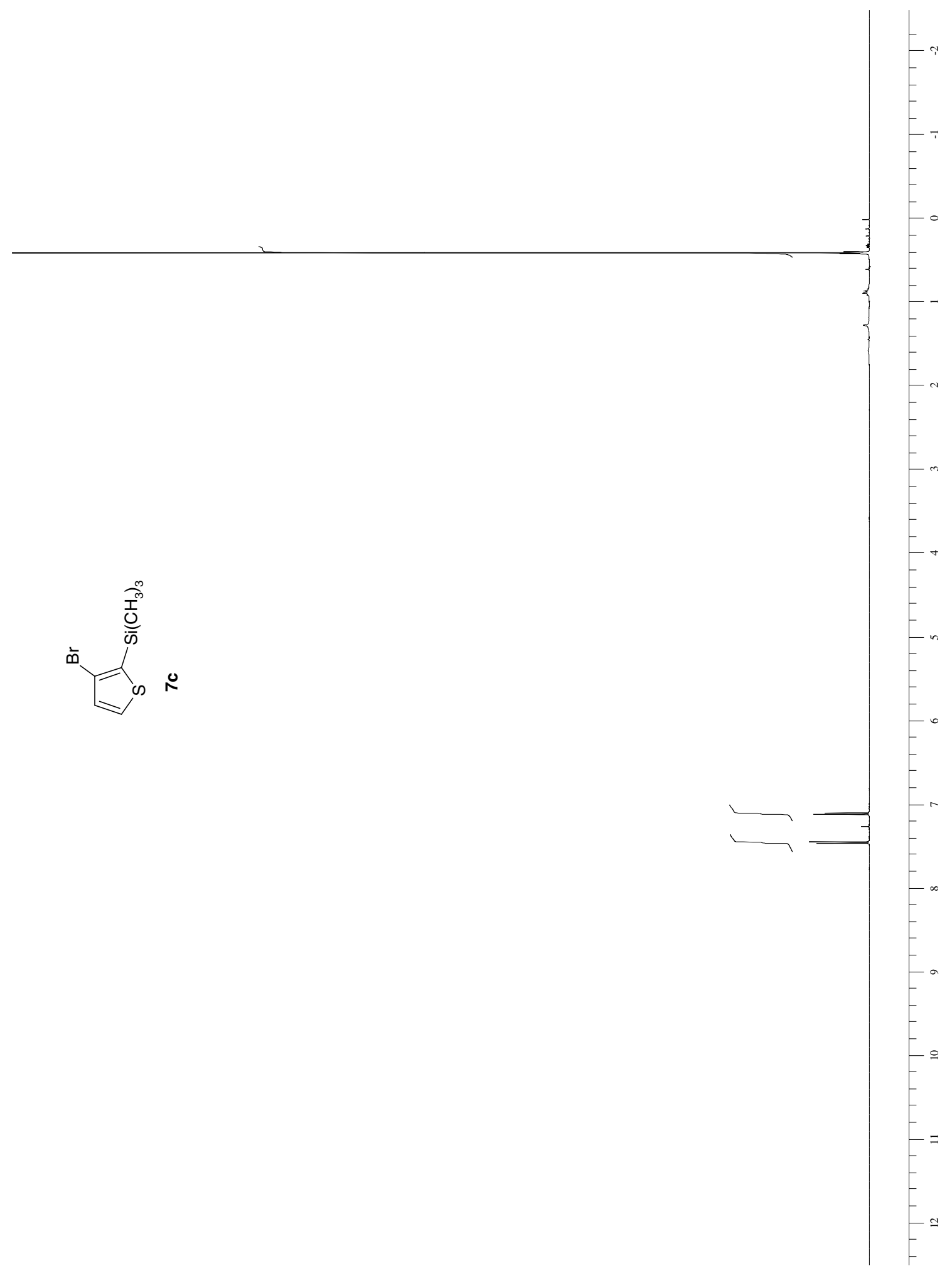




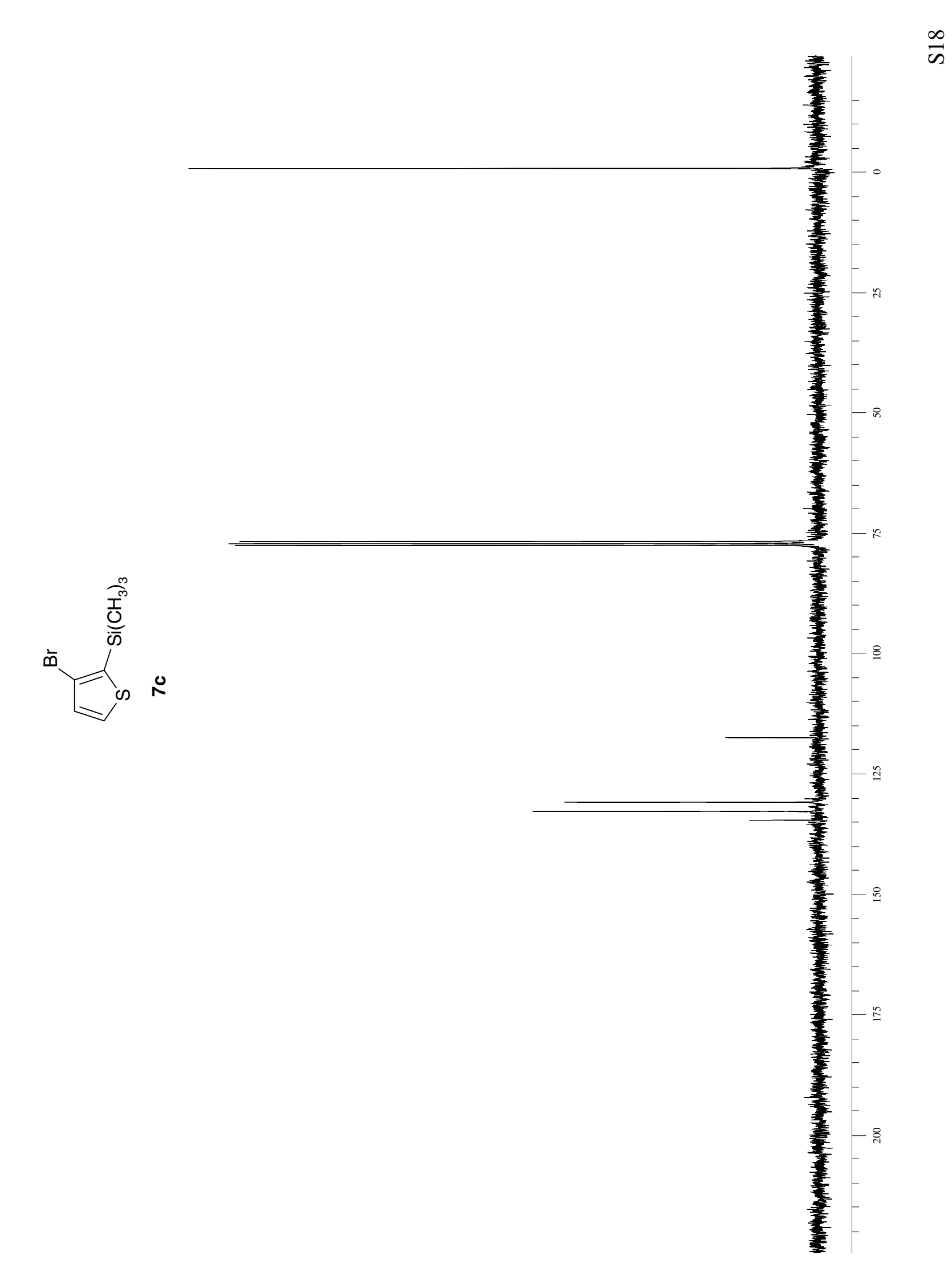




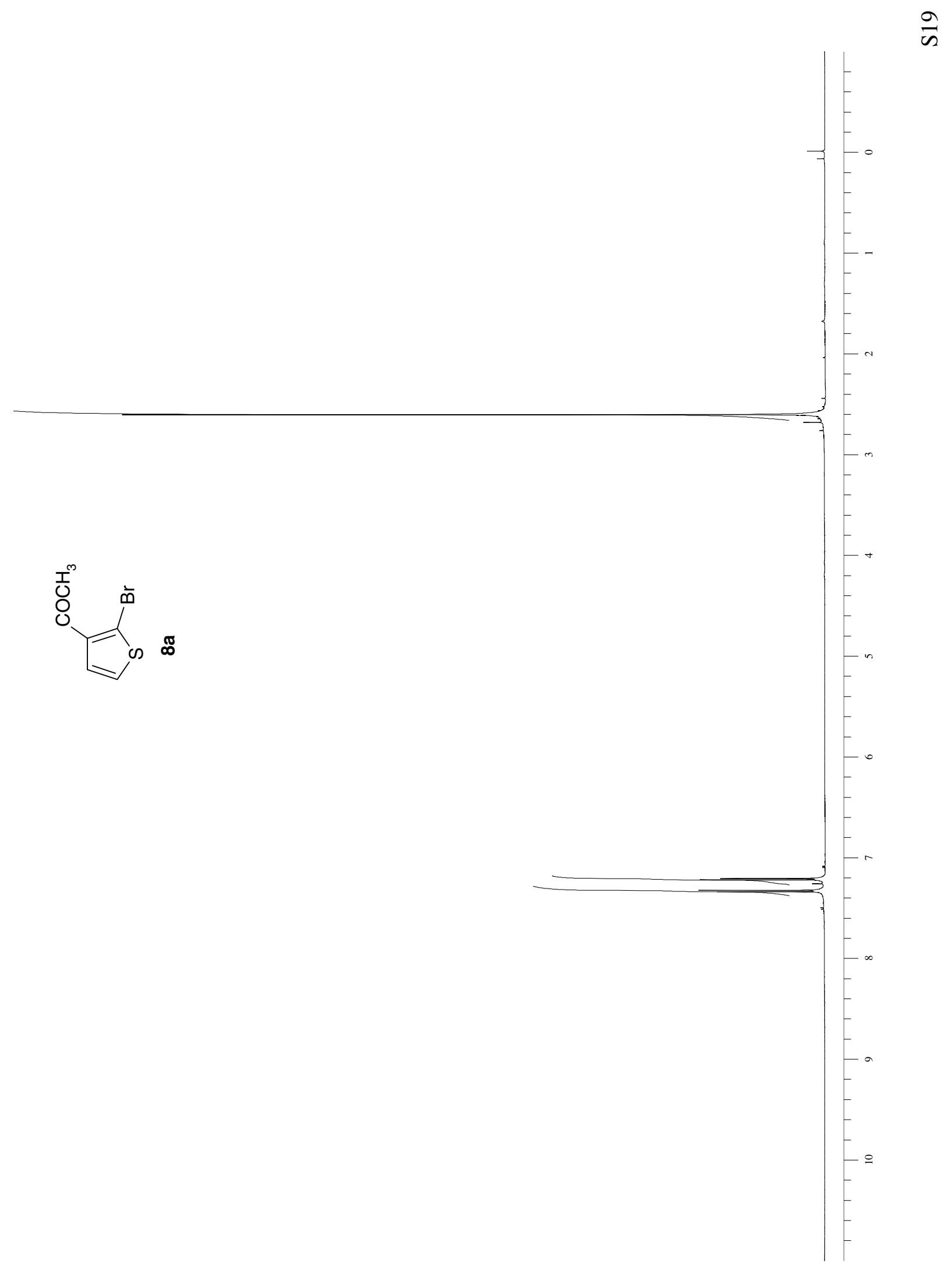




$$
1
$$




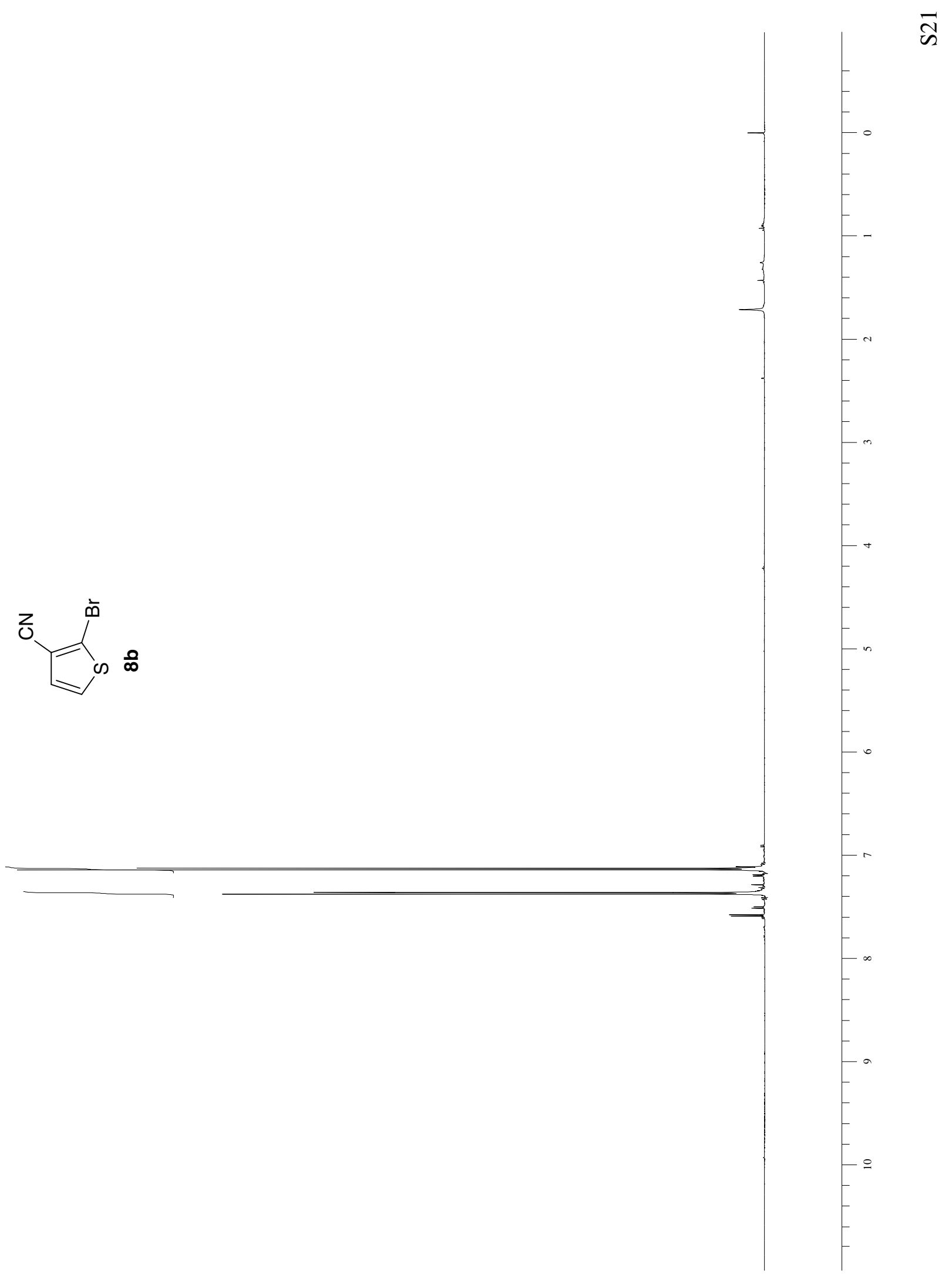




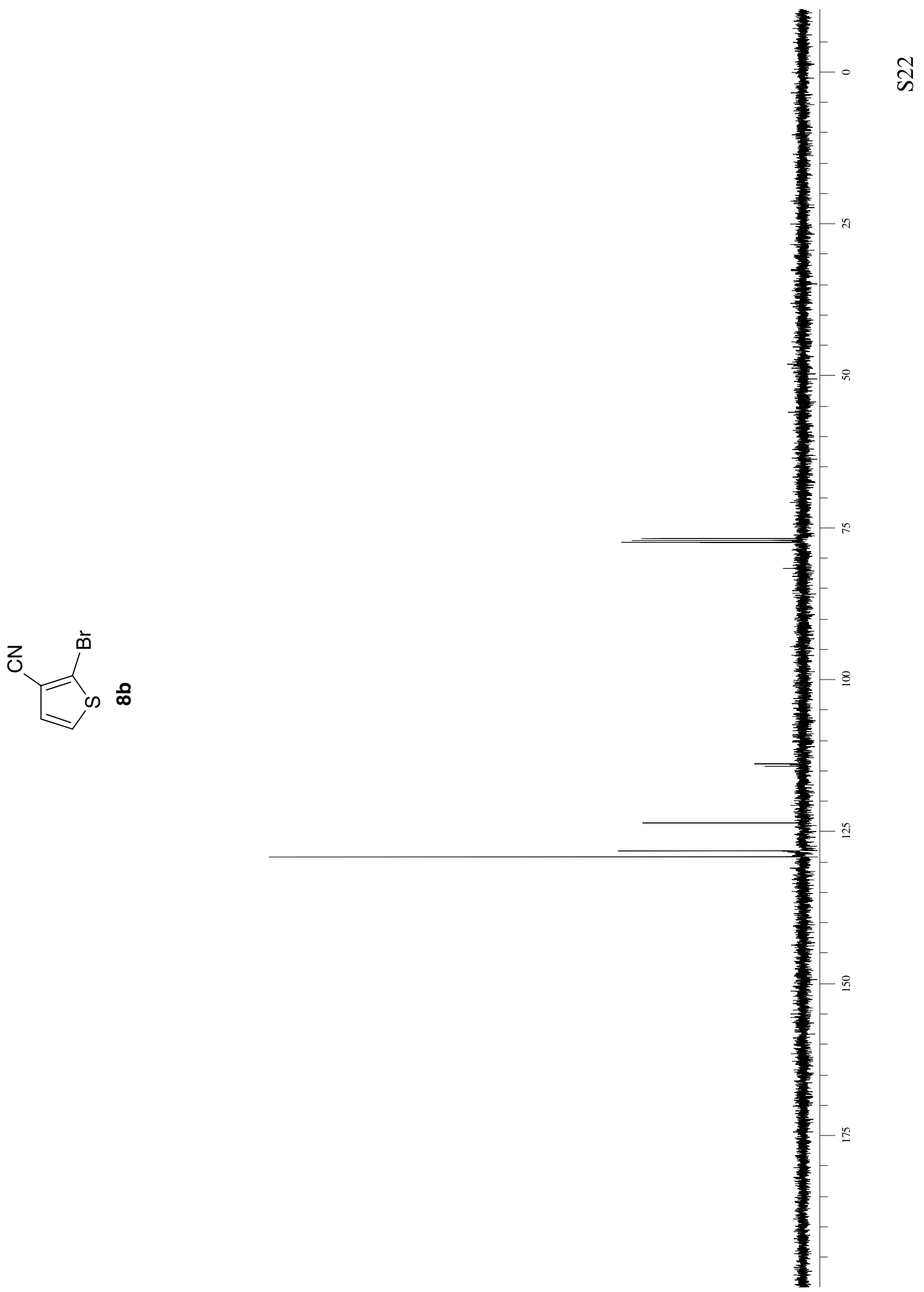




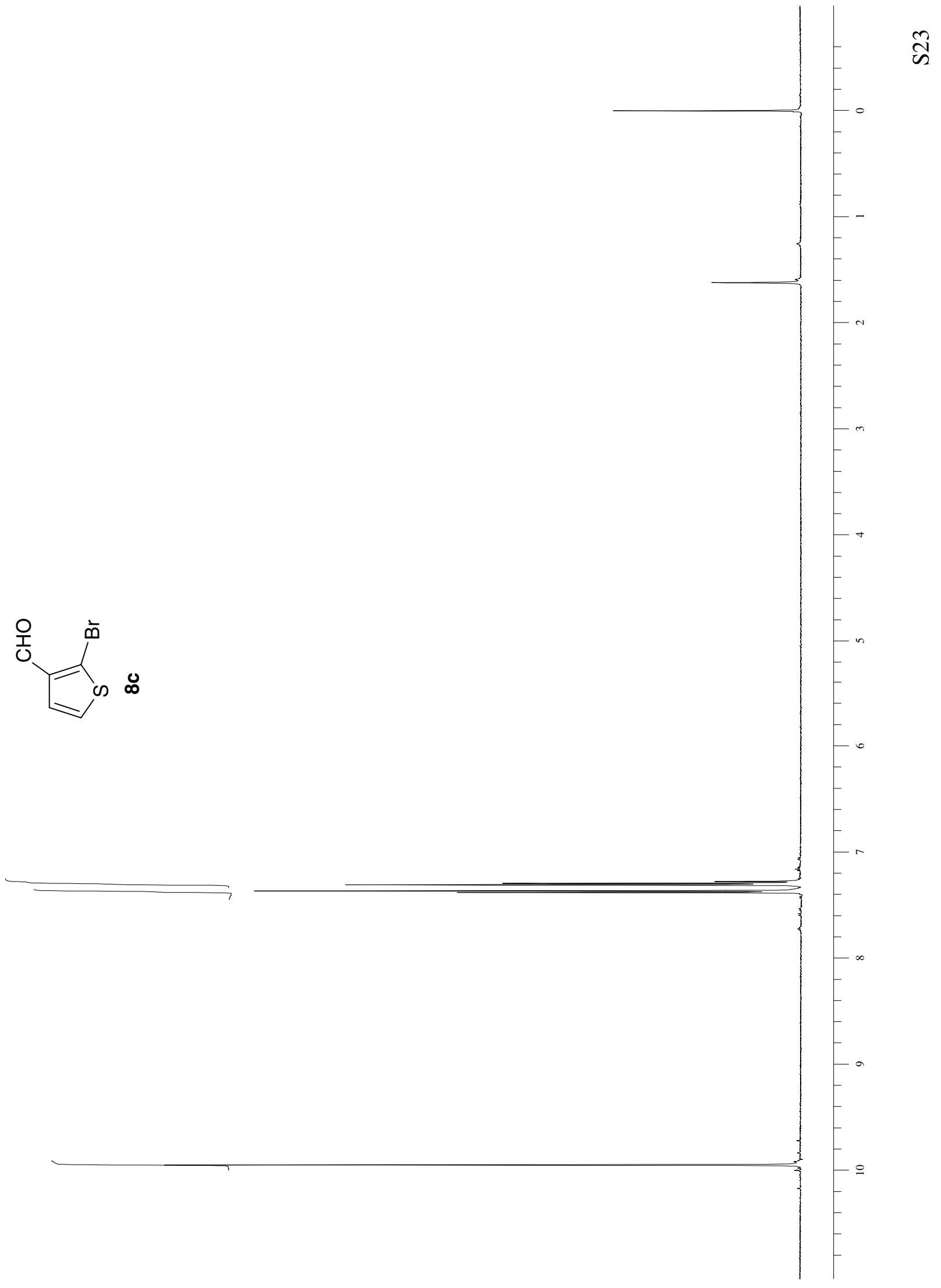




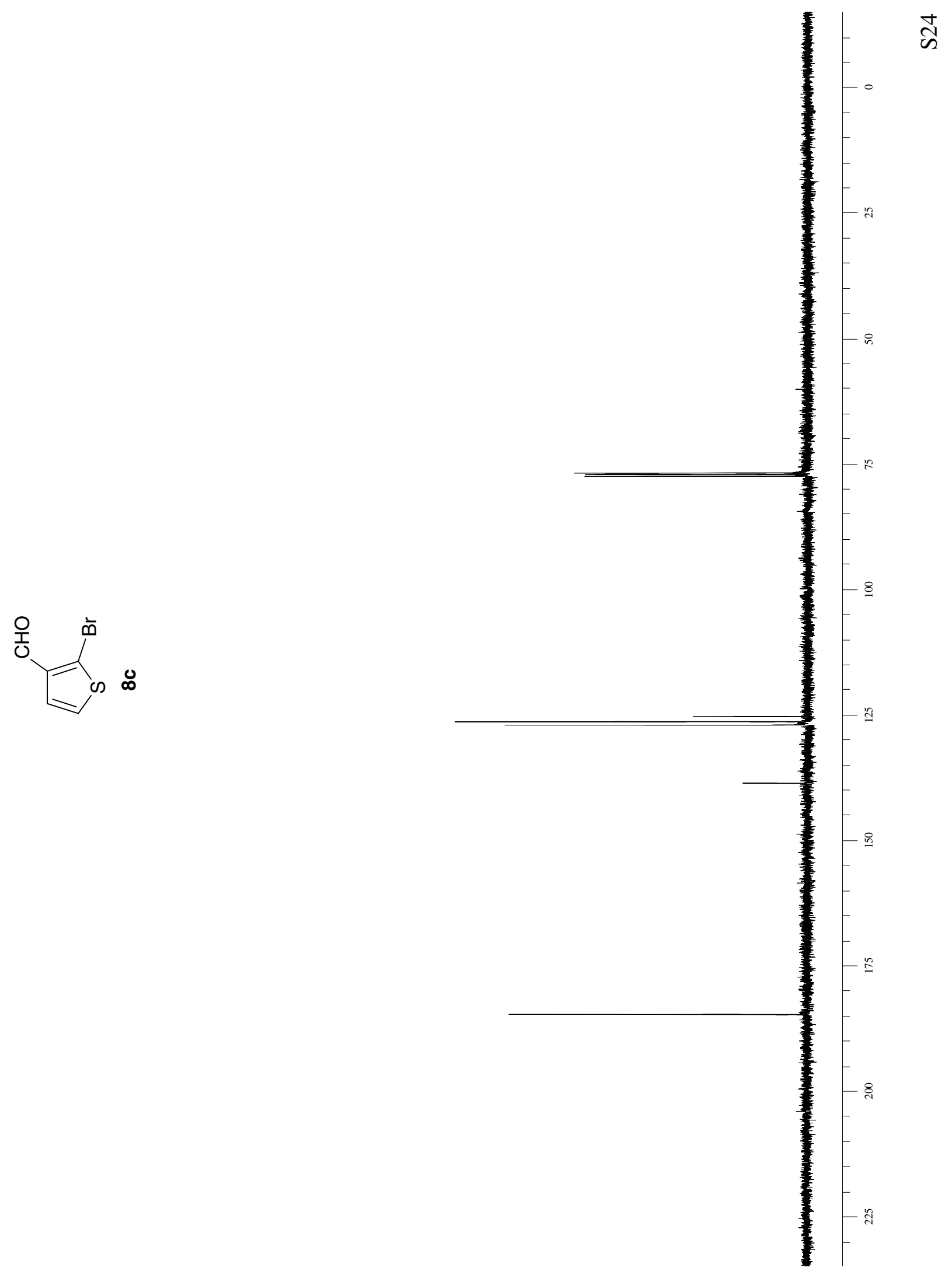




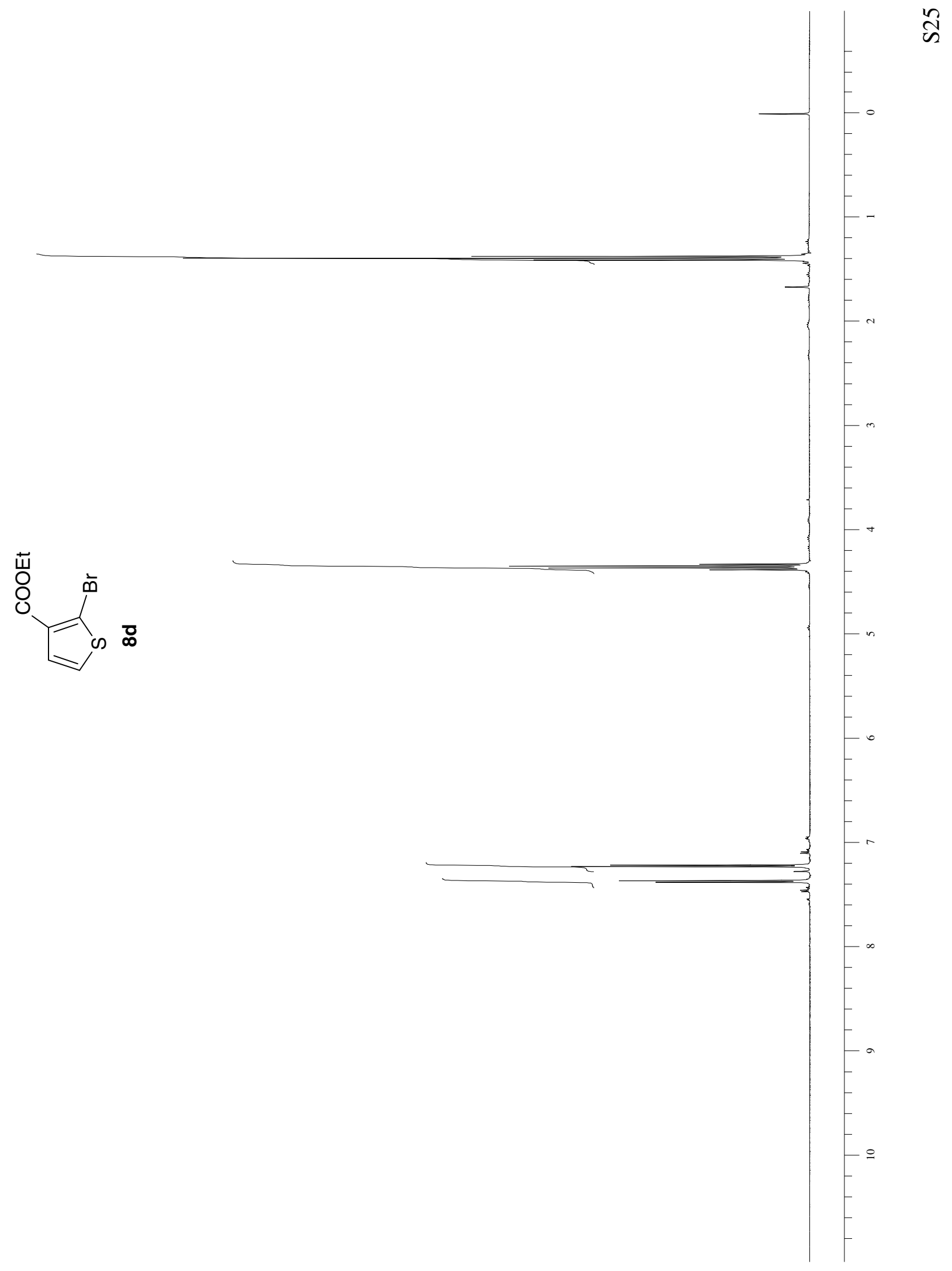




$$
\cdot 1
$$




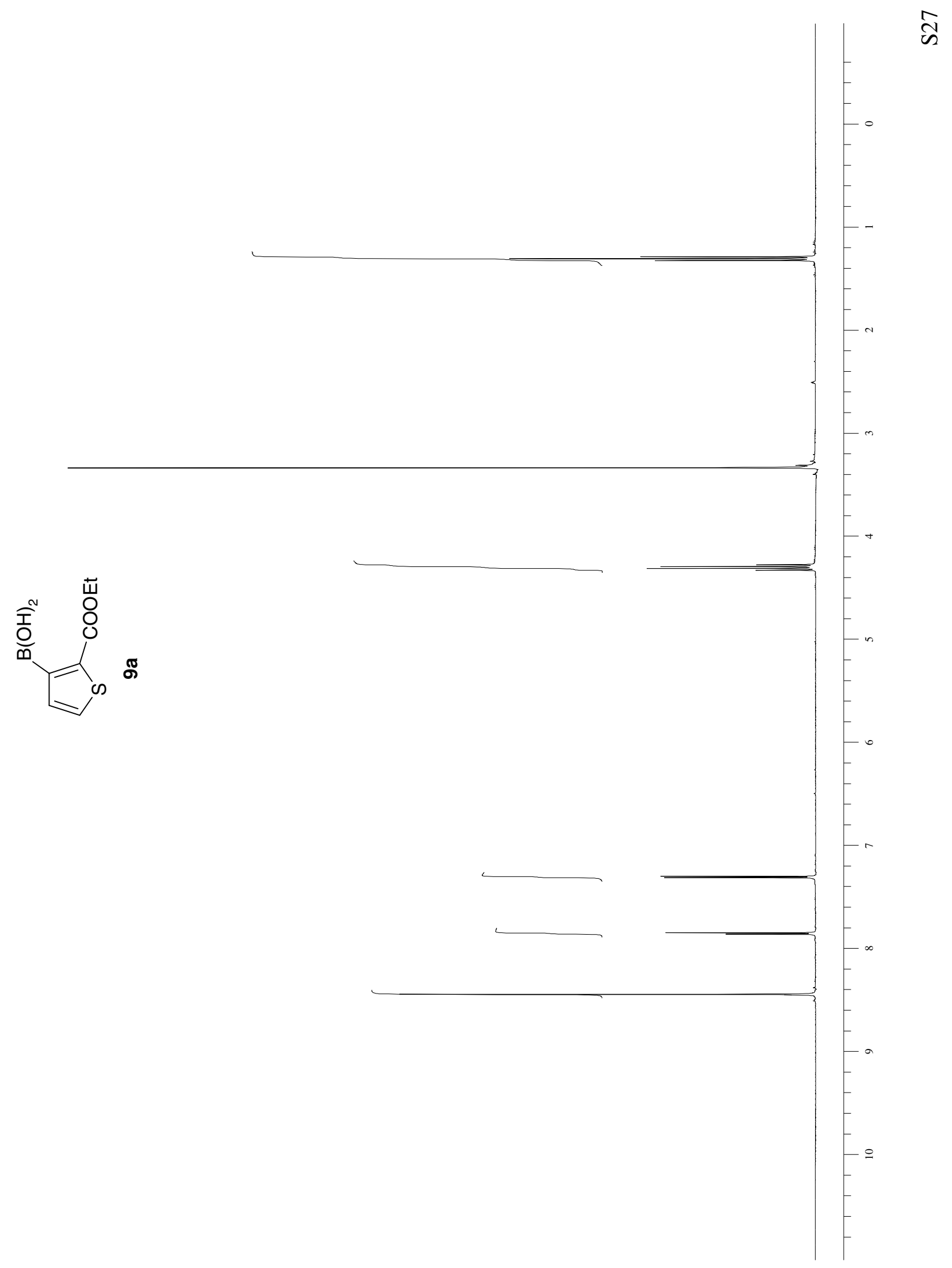




$$
1
$$




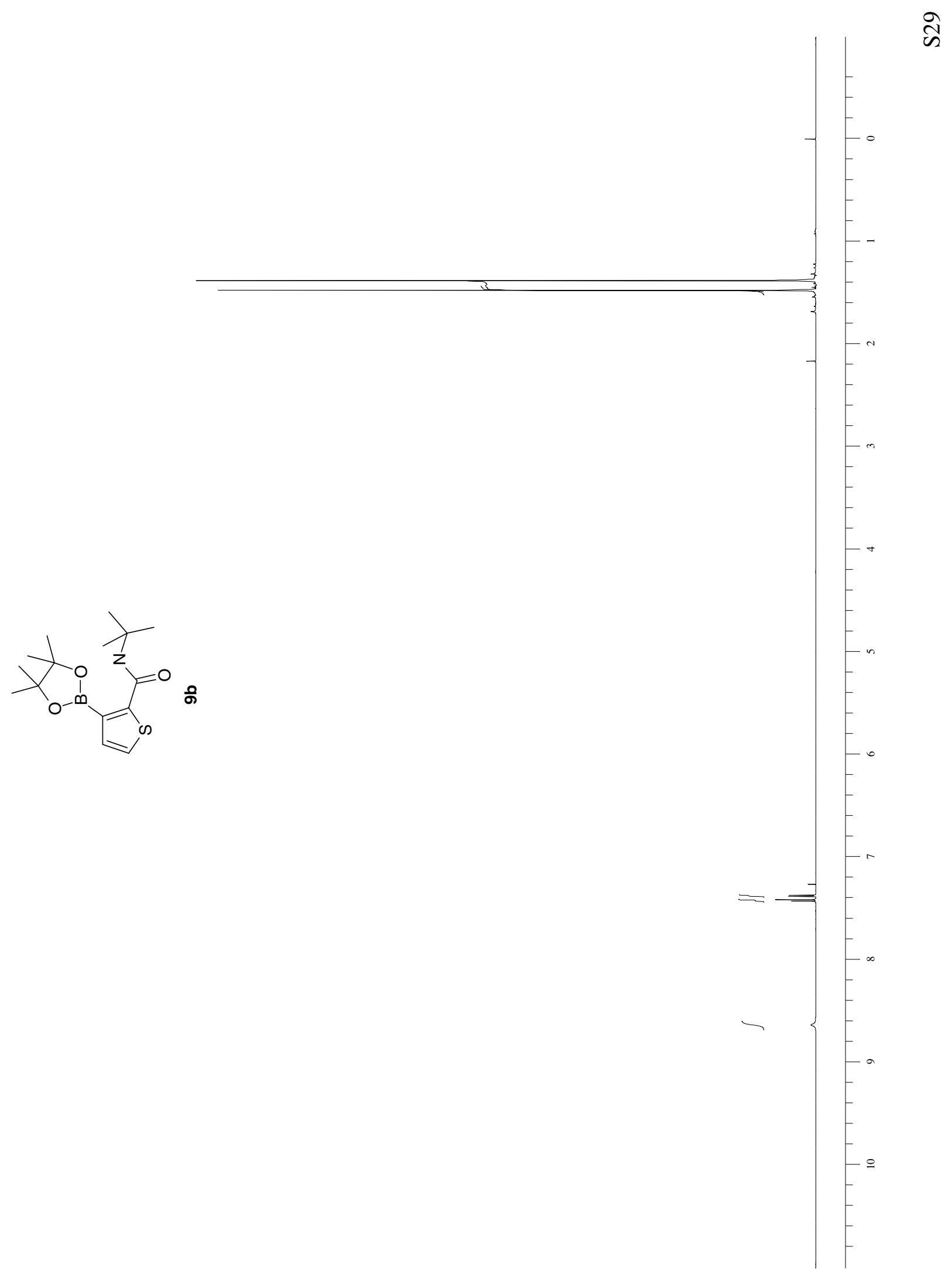




$$
.1
$$




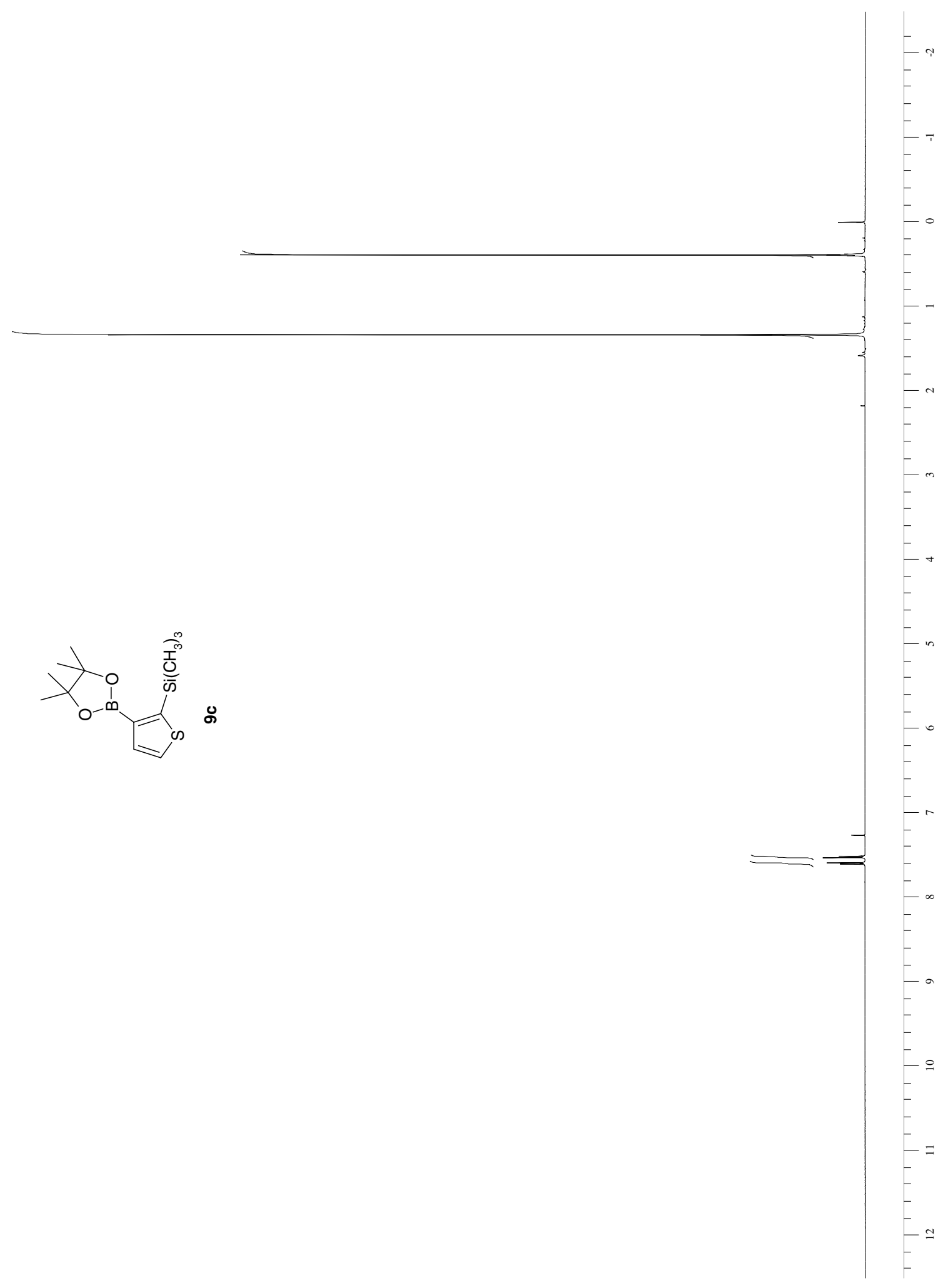




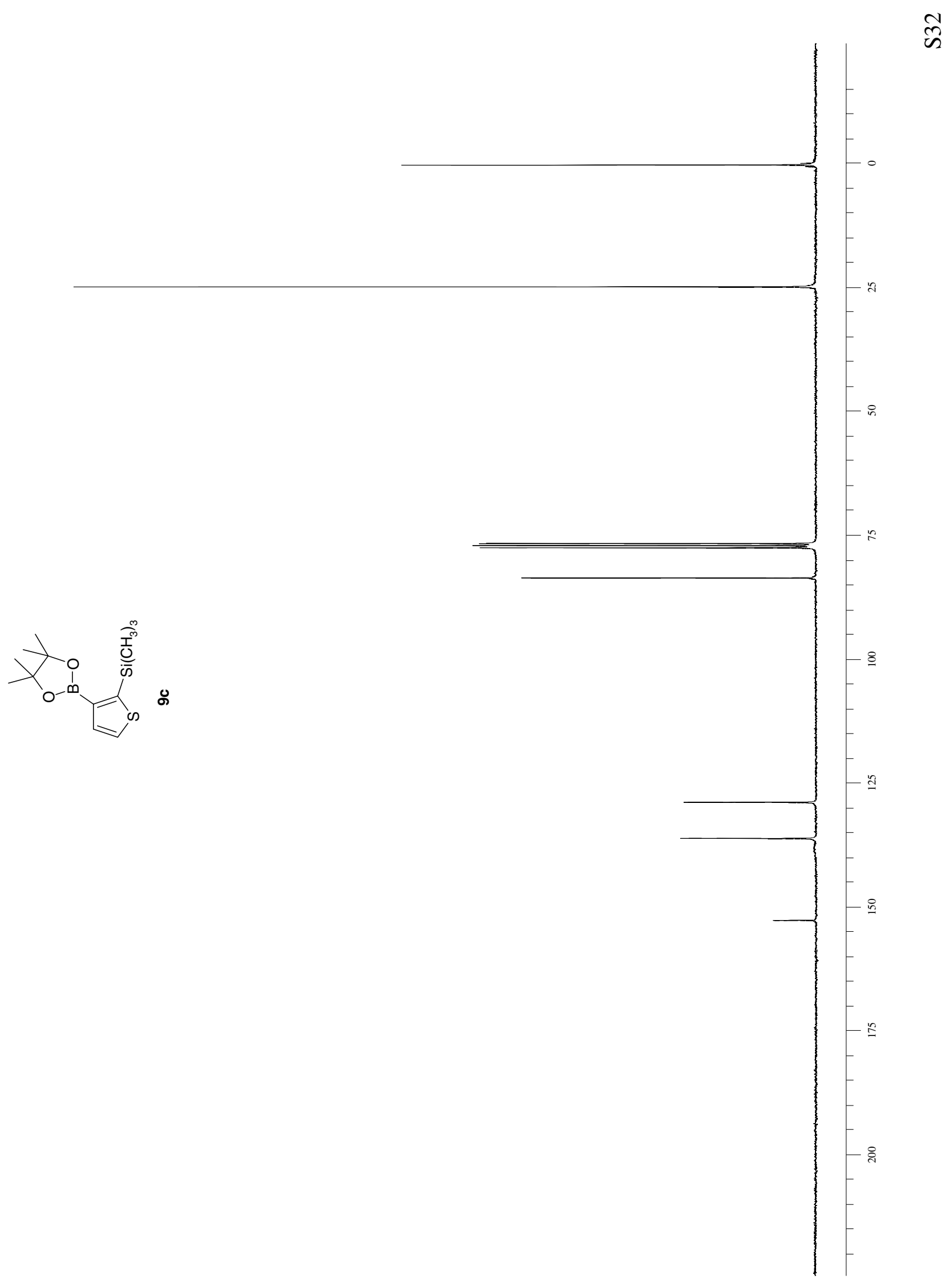




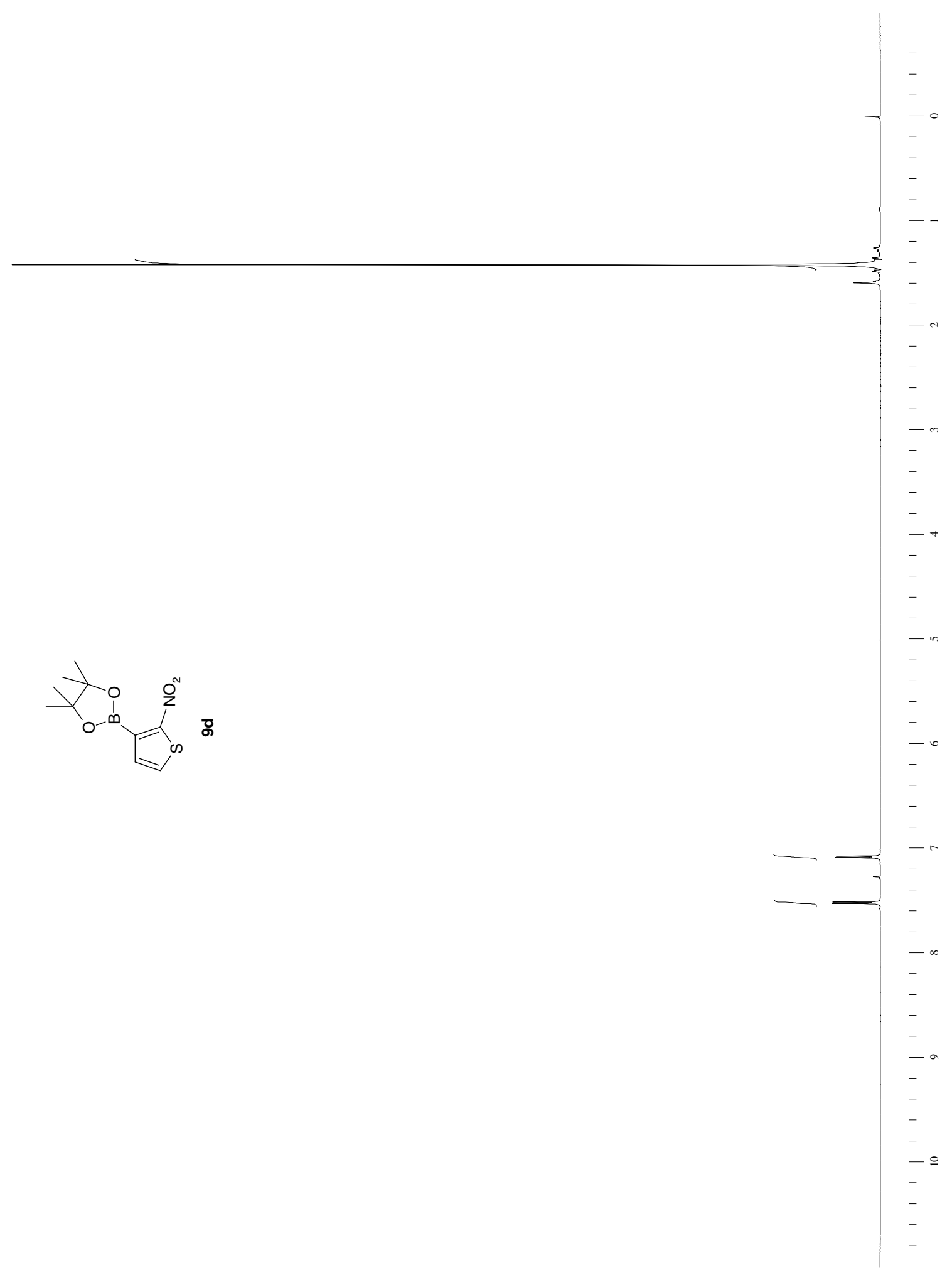




$$
1
$$




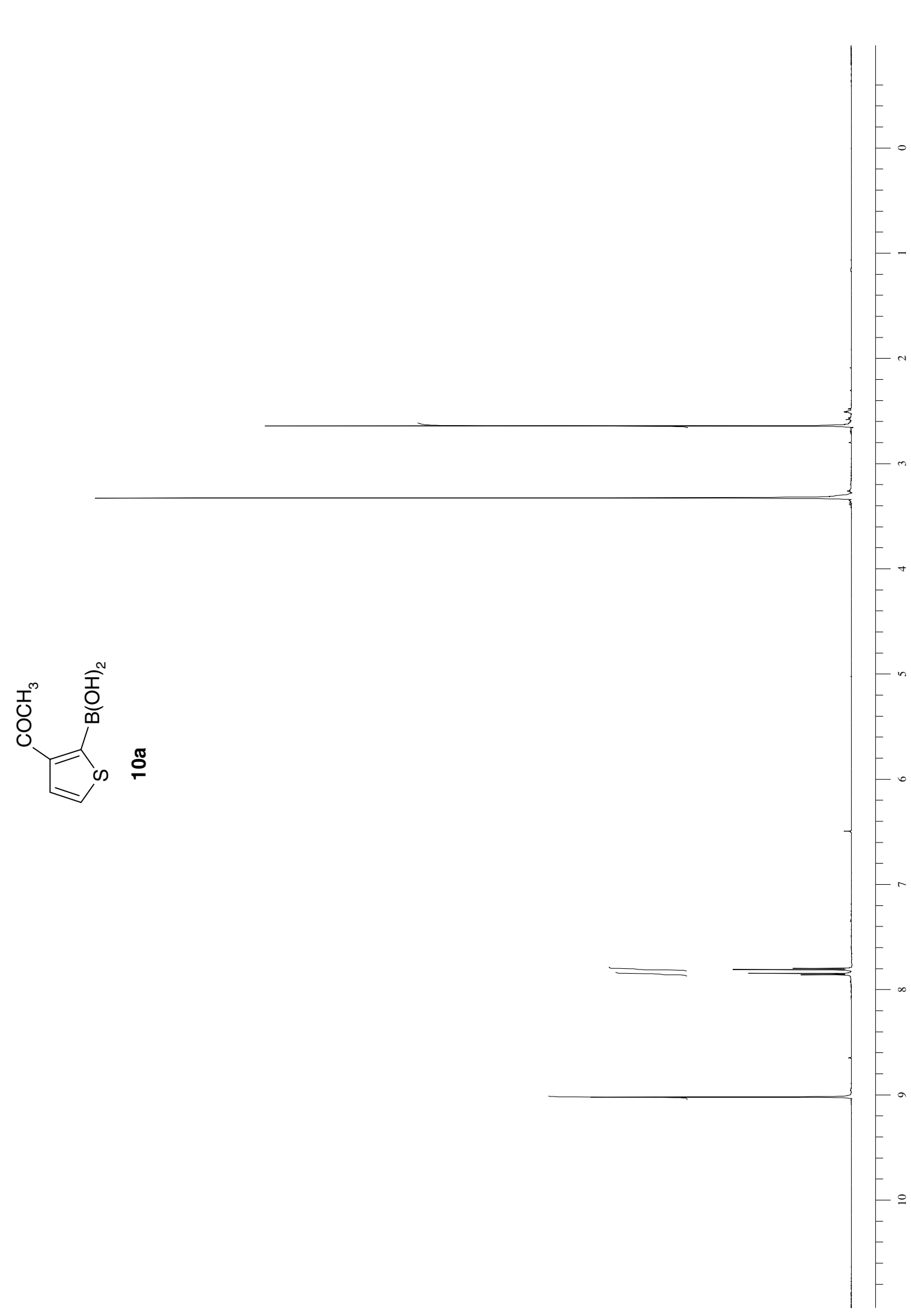




$$
1
$$


n

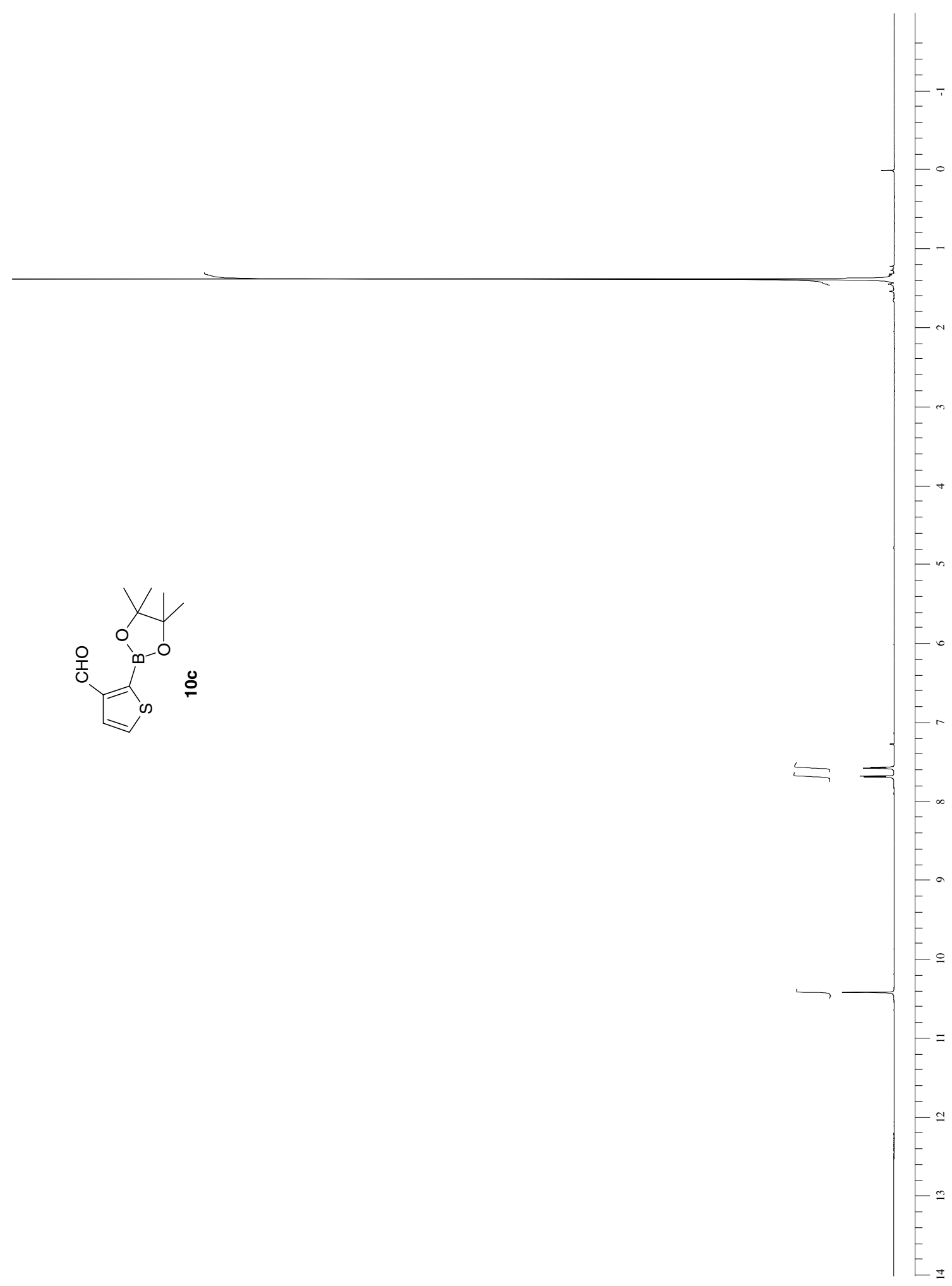




$$
\text { |' }
$$




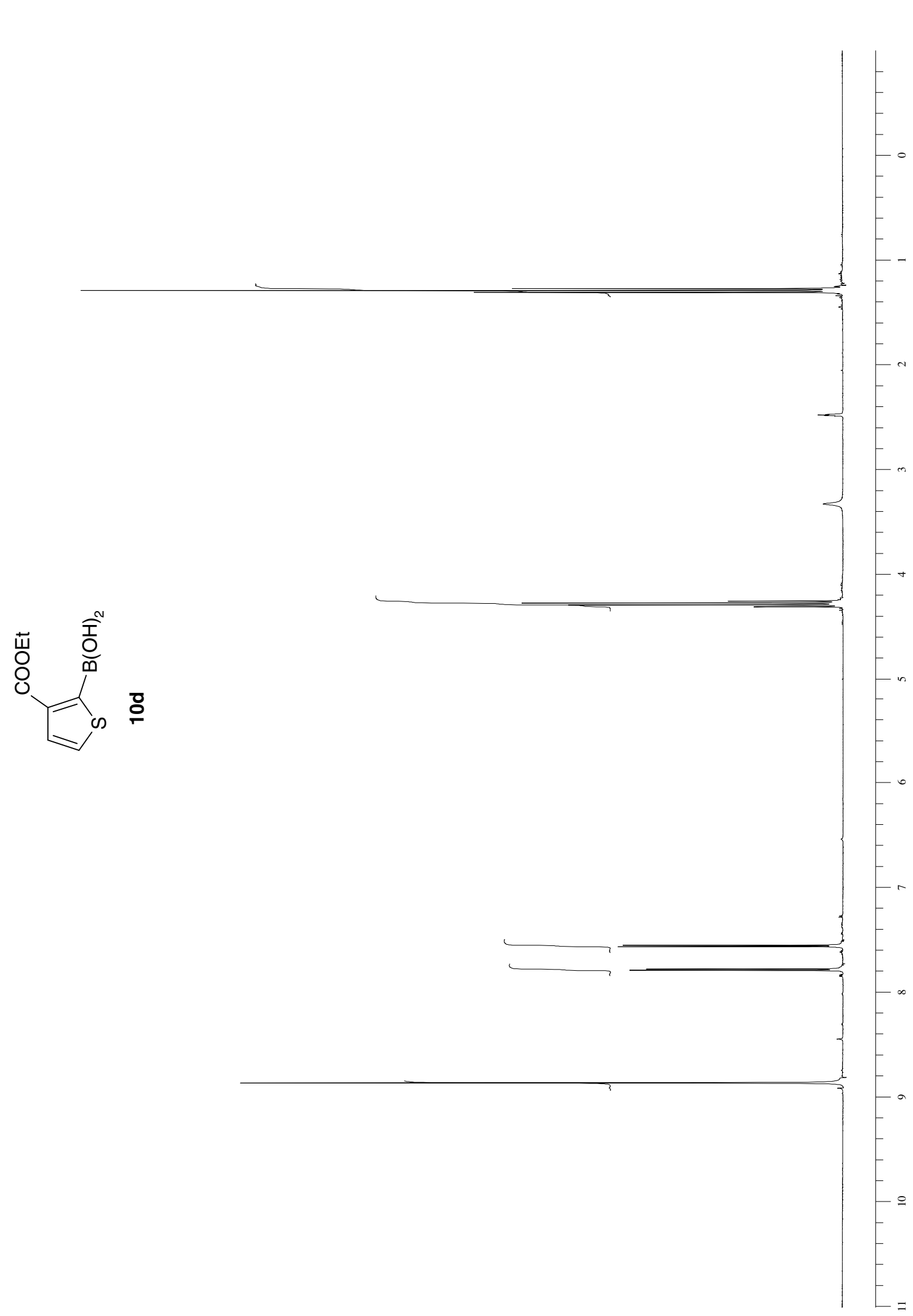

ळิ 


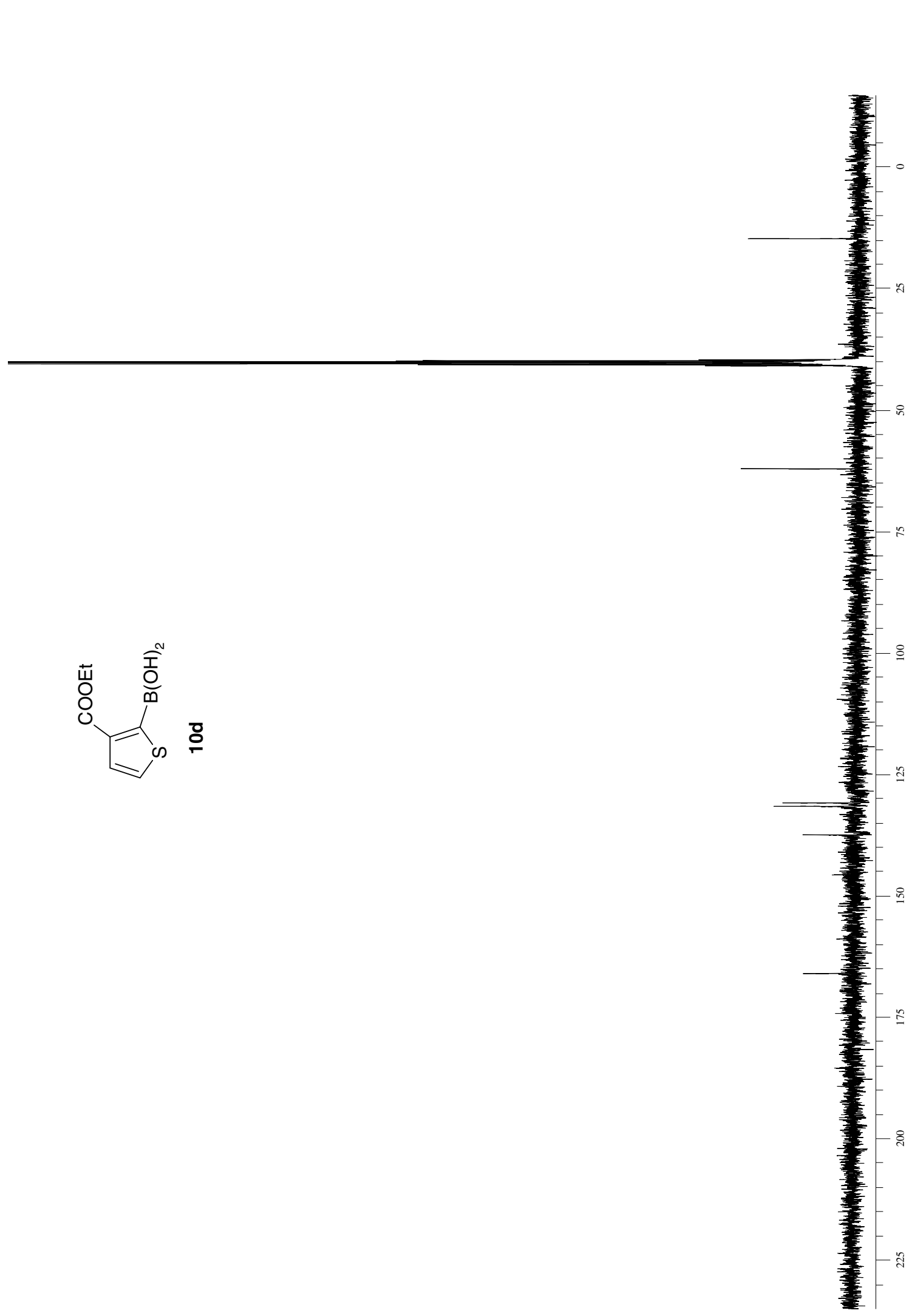

ษ 


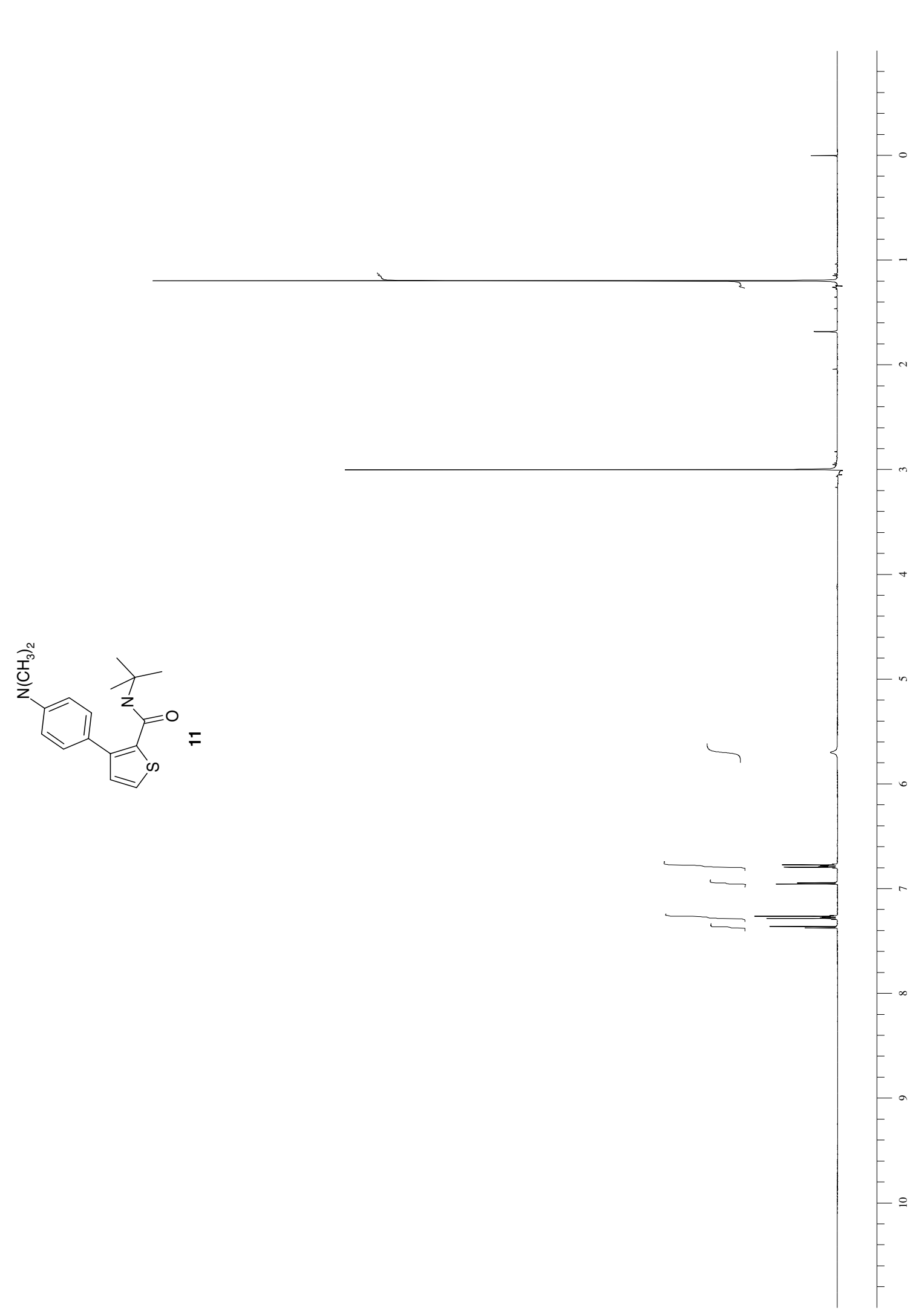

F 


$$
1
$$

